\title{
Resolved 24.5 micron emission from massive young stellar objects ${ }^{\star}$
}

\author{
W. J. de Wit ${ }^{1}$, M. G. Hoare ${ }^{1}$, T. Fujiyoshi ${ }^{2}$, R. D. Oudmaijer ${ }^{1}$, M. Honda ${ }^{3}$, H. Kataza ${ }^{4}$, T. Miyata ${ }^{5}$, Y. K. Okamoto ${ }^{6}$, \\ T. Onaka ${ }^{7}, \mathrm{~S}_{\text {. Sako }}{ }^{5}$, and T. Yamashita ${ }^{2}$ \\ 1 School of Physics \& Astronomy, University of Leeds, Woodhouse Lane, Leeds LS2 9JT, UK \\ e-mail: w.j.m.dewit@leeds.ac.uk \\ 2 Subaru Telescope, National Astronomical Observatory of Japan, National Institutes of Natural Sciences, 650 North A'ohoku Place, \\ Hilo, HI 96720, USA \\ 3 Department of Information Science, Kanagawa University, 2946 Tsuchiya, Hiratsuka, Kanagawa, 259-1293, Japan \\ 4 Department of Infrared Astrophysics, Institute of Space and Astronautical Science, Japan Aerospace Exploration Agency, \\ Sagamihara, Kanagawa, 229-8510, Japan \\ 5 Institute of Astronomy, University of Tokyo, Osawa 2-21-1, Mitaka, Tokyo 181-0015, Japan \\ ${ }^{6}$ Faculty of Science, Ibaraki University, 2-1-1 Bunkyo, Mito, Ibaraki 310-8512, Japan \\ 7 Department of Astronomy, Graduate School of Science, University of Tokyo, Bunkyo-ku, Tokyo 113-0022, Japan
}

Received 8 August 2008 / Accepted 24 November 2008

\begin{abstract}
Context. Massive young stellar objects (MYSO) are surrounded by massive dusty envelopes, whose physical structure and geometry are determined by the star formation process.

Aims. Our principal aim is to establish the density structure of MYSO envelopes on scales of $\sim 1000$ AU. This constitutes an increase of a factor $\sim 10$ in angular resolution compared to similar studies performed in the (sub)mm.

Methods. We have obtained diffraction-limited (0.6") $24.5 \mu \mathrm{m}$ images (field of view of $40^{\prime \prime} \times 30^{\prime \prime}$ ) of 14 well-known massive star formation regions with the COMICS instrument mounted on the $8.2 \mathrm{~m}$ Subaru telescope. We construct azimuthally averaged intensity profiles of the resolved MYSO envelopes and build spectral energy distributions (SEDs) from archival data and the COMICS $24.5 \mu \mathrm{m}$ flux density. The SEDs range from near-infrared to millimeter wavelengths. Self-consistent 1-D radiative transfer models described by a density dependence of the form $n(r) \propto r^{-p}$ are used to simultaneously compare the intensity profiles and SEDs to model predictions.

Results. The images reveal the presence of discrete MYSO sources which are resolved on arcsecond scales, and, to first-order, the observed emission is circular on the sky. For many sources, the spherical models are capable of satisfactorily reproducing the $24.5 \mu \mathrm{m}$ intensity profile, the $24.5 \mu \mathrm{m}$ flux density, the $9.7 \mu \mathrm{m}$ silicate absorption feature, and the submm emission. They are described by density distributions with $p=1.0 \pm 0.25$. Such distributions are shallower than those found on larger scales probed with single-dish (sub)mm studies. Other sources have density laws that are shallower/steeper than $p=1.0$ and there is evidence that these are viewed near edge-on or near face-on respectively. In these cases spherical models fail to provide good fits to the data. The images also reveal a diffuse component tracing somewhat larger scale structures, particularly visible in the regions S 140, AFGL 2136, IRAS 20126+4104, Mon R2, and Cep A.

Conclusions. We find a flattening of the MYSO density law going from scales probed with single-dish submm observations down to scales of $\sim 1000$ AU probed with the observations presented here. We propose that this may be evidence of rotational support of the envelope. This finding will be explored further in a future paper using 2-D axisymmetric radiative transfer models.
\end{abstract}

Key words. stars: formation - stars: imaging - stars: early-type - infrared: stars - ISM: clouds

\section{Introduction}

The early stages in the lives of massive stars are identified with highly luminous yet very cool objects deeply embedded in molecular clouds. Their emission is characterised by a steeply rising spectral energy distribution (SED) peaking around $100 \mu \mathrm{m}$, which features strong silicate absorption. These properties are indicative of radiation reprocessed by a dusty circumstellar envelope. Measured bolometric luminosities are such that if a single main sequence star resides at the heart of the dusty envelope, it should have the ability to ionise its surroundings, yet only little (if any) recombination radiation is observed. This

$\star$ Based on data collected at the Subaru telescope, which is operated by the National Astronomical Observatory of Japan. radio-quiet appearance of massive young stellar objects (MYSO) is unlike that of ultra-compact (UC) H II regions (e.g. Hoare et al. 2007), and the latter can therefore be considered as a successor phase. The reason may be that the MYSO is actively accreting material from its surrounding environment quenching the development of an $\mathrm{H}$ II region. The ongoing accretion would also give rise to bipolar outflow activity ubiquitously observed in massive star forming regions. It is clear that MYSOs are prime observational targets for addressing outstanding issues in our current understanding of massive star formation.

In this paper we address the radial structure of the MYSO dust envelope. It is determined by the forces that operate during the onset and the subsequent evolution of the initial molecular core. The radial density profile is predicted to be a powerlaw 
with a value for the power index that depends on the dominant physics. The exact power index can be extracted from the observables by the use of radiative transfer models. Spherical envelope models may be assumed for the dust that dominates the emission at wavelengths larger than $30 \mu \mathrm{m}$. At these wavelengths the SED of MYSOs (but also UCH IIs) are observed to be remarkably similar, arguing for little deviation from spherical symmetry (Chini et al. 1986; Churchwell et al. 1990; Henning et al. 1990; Hoare et al. 1991; Gürtler et al. 1991; Wolfire \& Churchwell 1994; Faison et al. 1998; Hatchell et al. 2000; Van der Tak et al. 2000; Mueller et al. 2002; Beuther et al. 2002). At shorter wavelengths $(\lambda<30 \mu \mathrm{m})$ the geometry of the envelope becomes important (Yorke \& Sonnhalter 2002; Whitney et al. 2003). For example, under favourable inclinations, mid-IR radiation can be observed to originate directly from the surface of cavity walls that are sculpted by the polar outflows. In this case, the mid-IR photons are emitted by warm dust particles that have a clear line-of-sight to the star (e.g. De Buizer 2007). At even shorter wavelengths, near-IR photons from the (generally monopolar) reflection nebulae (e.g. Alvarez et al. 2004, 2005) may originate either from the stellar surface, an inner dust truncation structure or from an accretion disc. They can scatter and escape through existing inhomogeneities in the spherical envelope (e.g. Gürtler et al. 1991; Henning et al. 2000) and still suffer extinction from any foreground molecular cloud material (e.g. De Buizer et al. 2008).

Here, we aim to constrain the radial density distribution on scales of $1000 \mathrm{AU}$ using resolved $24.5 \mu \mathrm{m}$ emission. This constitutes an increase of a factor 10 in angular resolution compared to similar studies performed in the (sub)mm. We present diffraction-limited images at $24.5 \mu \mathrm{m}$, the longest mid-IR wavelength amenable to high resolution imaging from the ground with large telescopes. This long mid-IR wavelength maximises the possibility of resolving the envelope emission because, due to the nature of the temperature gradients in the optically thick emitting region, the size of the emission region gets larger with increasing wavelength to the power of about 1.5, whilst the diffraction limit of the telescope only increases linearly with wavelength. We have selected a set of 14 well-known MYSO and imaged these at $24.5 \mu \mathrm{m}$ with the $8 \mathrm{~m}$ Subaru telescope. The images have an angular resolution set by the telescope's diffraction limit of $0.6^{\prime \prime}$, corresponding to linear scales of $\sim 1000 \mathrm{AU}$ for the average distance of $1.5 \mathrm{kpc}$ to our target MYSOs. Most previous $10 \mu \mathrm{m}$ and $20 \mu \mathrm{m}$ imaging of MYSOs have been carried out on $4 \mathrm{~m}$ class telescopes where the (radio-quiet) objects are invariably unresolved (e.g. Mottram et al. 2007; De Buizer et al. 2000, 2008). Some work on 8-10 m class telescopes at 8-22 $\mu \mathrm{m}$ has begun to resolve a few sources such as BN and source $n$ in Orion (Shuping et al. 2004; Greenhill et al. 2004), but no detailed modelling of these data has been carried out.

We analyse the resolved emission in conjunction with the SED in terms of spherical dust radiative transfer models as calculated by DUSTY, and using background literature information for each individual case. We present simultaneous model fits to the $24.5 \mu \mathrm{m}$ intensity profile and the SED, that stretches from the near-IR to the mm wavelengths. It includes the silicate $9.7 \mu \mathrm{m}$ absorption profile thanks to ISO-SWS spectra for nearly the whole sample. The approach of simultaneously analysing the intensity profiles and SEDs follows van der Tak et al. (2000); Hatchell et al. (2000); Beuther et al. (2002); Mueller et al. (2002); Hatchell \& van der Tak (2003); Williams et al. (2005). Most of the quoted work is exclusively aimed at (sub)mm wavelengths, and thus probing linear scales about a factor 10 larger than in the mid-IR. Constraints on the density distributions from
Table 1. COMICS $24.5 \mu \mathrm{m}$ observing log of standard stars ( $\mathrm{P}=\mathrm{PSF}$ standard; $\mathrm{S}=$ standard). Standard stars are further divided into Cohen (C) and Sloan (L) standards. Upper part of the table are observations performed with the $Q 24.5$ - OLD filter, the lower part with the $Q 24.5$ $N E W$ filter. Filter characteristics are given in Table 3.

\begin{tabular}{ccrrr}
\hline \hline $\begin{array}{c}\text { Date } \\
(\mathrm{UT})\end{array}$ & Object & $\begin{array}{r}\text { Integration } \\
(\mathrm{s})\end{array}$ & $\overline{A M}$ & $S / N$ \\
\hline $2002 / 12 / 15$ & Asteroid \#511 (P) & 502 & 1.8 & 900 \\
$2002 / 12 / 15$ & Asteroid \#51 (P) & 402 & 2.0 & 160 \\
$2003 / 06 / 17$ & $\mu$ Cep (SL) & 402 & 1.3 & 1800 \\
$2003 / 06 / 20$ & $\mu$ Cep (SL) & 402 & 1.3 & 1000 \\
$2003 / 07 / 14$ & $\mu$ Cep (SL) & 101 & 1.5 & 180 \\
$2003 / 11 / 12$ & $\alpha$ Tau (SL) & 1204 & 1.2 & 800 \\
$2004 / 01 / 08$ & $\alpha$ Tau (SL) & 402 & 1.0 & 830 \\
$2004 / 05 / 05$ & $\mu$ Cep (SL) & 201 & 1.4 & 790 \\
\hline $2004 / 06 / 08$ & $\alpha$ Her (SL) & 100 & 1.2 & 630 \\
$2004 / 06 / 08$ & $\mu$ Cep (SL) & 602 & 1.3 & 3000 \\
$2004 / 06 / 08$ & $\alpha$ Sco (SL) & 201 & 1.9 & 1200 \\
$2004 / 07 / 14$ & $\mu$ Cep (SL) & 401 & 1.3 & 1500 \\
$2005 / 07 / 27$ & $\alpha$ Her (SL) & 201 & 2.6 & 160 \\
$2005 / 07 / 27$ & $\gamma$ Aql (SC) & 201 & 1.3 & 30 \\
$2005 / 12 / 13$ & $\alpha$ Tau (SL) & 401 & 1.3 & 430 \\
$2005 / 12 / 21$ & $\alpha$ Tau (SL) & 1203 & 1.4 & 620 \\
\hline
\end{tabular}

(sub)mm and mid-IR are therefore highly complementary as they give insight into the evolution of the density distribution as function of radius.

This paper is organized as follows. Our observational data were taken with the Japanese $8.2 \mathrm{~m}$ Subaru telescope on Hawaii in conjunction with the COMICS instrument. We give an overview of the instrument and detail the observations in Sect. 2. We present and discuss the morphology seen on the COMICS images in the subsections to Sects. 3.3 and 3.4, alongside the simultaneous modelling of the intensity profile and SED. We summarise the modelling part highlighting certain trends in Sect. 4. We discuss our findings and their consequences in Sect. 5 and conclude in Sect. 6.

\section{Observations and data reduction}

\subsection{Observations with the COMICS instrument}

Tables 1 and 2 summarise the $24.5 \mu \mathrm{m}$ observations of the 14 target MYSOs and the standard stars. The signal to noise in Col. 5 of Table 2 is calculated as peak flux divided by the background standard deviation.

All measurements were made using the mid-infrared imaging spectrometer COMICS (Kataza et al. 2000) at the Cassegrain focus of the $8.2 \mathrm{~m}$ Subaru Telescope on Mauna Kea, Hawaii. Imaging mode of COMICS utilises a Raytheon $320 \times 240$ Si:As IBC array, which is cooled by a Sumitomo 4-K GiffordMcMahon type cryo-cooler but usually operates at around $7 \sim$ $8 \mathrm{~K}$ because of the self-heating. The camera provides oversampled diffraction-limited images at $24.5 \mu \mathrm{m}$ with a pixel size of $0.13 \times 0.13 \operatorname{arcsec}^{2}$ and a field of view of approximately $40 \times 30 \operatorname{arcsec}^{2}$.

We used two different $24.5 \mu \mathrm{m}$ filters which are both manufactured by Infrared Multilayer Laboratory, University of Reading. The characteristics of these filters are summarised in Table 3, and the transmission curves, along with an atmospheric transmission model above Mauna Kea, are shown in Fig. 1. As can be clearly seen in the plot, the new filter $(Q 24.5-N E W)$ is a much better fit to the small atmospheric window at $24.5 \mu \mathrm{m}$. As a result, the whole array can be read out with the new filter, 
Table 2. COMICS $24.5 \mu \mathrm{m}$ observing and image details. Observations after 2004/06/08 are performed with $Q 24.5-N E W$ filter. The J2000 coordinates correspond to the principal MYSO in each region, with their reference given in Col. 8. The offsets in Col. 9 correspond to the identification of known sources (Col. 10) in the images. The one uncertain identification is marked with a colon. The uncertainty of the flux densities given in Col. 11 is on the order of 10\%. The Mon R2 and W3 regions have been mosaiced using five, respectively four images (two long and two short exposures).

\begin{tabular}{|c|c|c|c|c|c|c|c|c|c|c|}
\hline $\begin{array}{l}\text { Region } \\
\text { (1) }\end{array}$ & $\begin{array}{l}\text { Date } \\
\text { (UT) } \\
\text { (2) }\end{array}$ & $\begin{array}{l}\text { Integration } \\
\text { (s) } \\
\text { (3) }\end{array}$ & $\begin{array}{l}\overline{\mathrm{AM}} \\
(4)\end{array}$ & $\begin{array}{l}S / N \\
(5)\end{array}$ & $\begin{array}{l}\text { RA } \\
\text { (h,m,s) } \\
(6)\end{array}$ & $\begin{array}{l}\text { Dec. } \\
\left({ }^{\circ},{ }^{\prime}, \prime\right) \\
(7)\end{array}$ & $\begin{array}{l}\text { Ref. } \\
\text { (8) }\end{array}$ & $\begin{array}{l}\text { Offset } \\
\left({ }^{\prime \prime}\right) \\
(9)\end{array}$ & $\begin{array}{l}\text { Source } \\
\text { ID } \\
(10)\end{array}$ & $\begin{array}{r}\text { Flux density } \\
(\mathrm{Jy}) \\
(11)\end{array}$ \\
\hline \multirow[t]{4}{*}{ S140 } & $2004 / 06 / 08$ & 602 & 1.4 & 2000 & $22: 19: 18.3$ & $+63: 18: 49.3$ & 1 & $(0,0)$ & IRS1 & 1170 \\
\hline & & & & & & & & $(2,14)$ & IRS2S & 5 \\
\hline & & & & & & & & $(0,18)$ & IRS2N: & 170 \\
\hline & & & & & & & & $(10,3)$ & IRS3 & 180 \\
\hline \multirow[t]{3}{*}{ M8E } & $2004 / 06 / 08$ & 802 & 1.4 & 1100 & 18:04:53.3 & $-24: 26: 42.3$ & 2 & $(0,0)$ & IR & 210 \\
\hline & & & & & & & & $(-6,5)$ & HII & 30 \\
\hline & & & & & & & & $(-6.5,0.5)$ & MIRS1 & 5 \\
\hline AFGL 2136 & $2005 / 07 / 27$ & 201 & 2.5 & 170 & $18: 22: 26.5$ & $-13: 30: 12.0$ & 3 & $(0,0)$ & IR & 140 \\
\hline \multirow[t]{3}{*}{ AFGL 2591} & $2004 / 05 / 05$ & 603 & 1.1 & 2000 & $20: 29: 24.9$ & $+40: 11: 20.3$ & 4 & $(0,0)$ & IR & 870 \\
\hline & & & & & & & & $(-5,-3)$ & HII & 170 \\
\hline & & & & & & & & $(-7,9)$ & MIRS1 & 20 \\
\hline NGC 2264 & $2002 / 12 / 15$ & 1306 & 1.9 & 1100 & $06: 41: 10.1$ & $+09: 29: 34.0$ & 5 & $(0,0)$ & IRS1 & 330 \\
\hline \multirow[t]{2}{*}{$\mathrm{S} 255$} & $2003 / 11 / 12$ & 1404 & 1.0 & 1000 & $06: 12: 54.1$ & $+17: 59: 25.1$ & 6 & $(0,0)$ & IRS3 & 170 \\
\hline & & & & & & & & $(-2.5,0.5)$ & IRS1 & 20 \\
\hline \multirow[t]{3}{*}{ AFGL 5180} & $2005 / 12 / 20$ & 1003 & 1.2 & 100 & 06:08:53.3 & $+21: 38: 30.5$ & 4 & $(0,0)$ & IRS1 & 490 \\
\hline & & & & & & & & $(12,-4)$ & HII & 210 \\
\hline & & & & & & & & $(2,2)$ & MIRS3 & 35 \\
\hline IRAS 20126 & $2005 / 07 / 27$ & 401 & 1.3 & 60 & $20: 14: 26.1$ & $+41: 13: 32.5$ & 7 & $(0,0)$ & IR & 60 \\
\hline \multirow[t]{3}{*}{ Mon R2 } & $2005 / 12 / 13$ & 100 & 1.2 & & 06:07:47.8 & $-06: 22: 54.7$ & 1 & $(0,0)$ & IRS3 & 1150 \\
\hline & & & & & & & & $(-31,3)$ & IRS2 & 40 \\
\hline & & & & & & & & $(-33,17)$ & IRS5 & 70 \\
\hline \multirow[t]{3}{*}{ AFGL 437} & $2005 / 12 / 21$ & 401 & 1.3 & 80 & 03:07:24.6 & $+58: 30: 44.4$ & 8 & $(0,0)$ & $\mathrm{S}$ & 30 \\
\hline & & & & & & & & $(0,10)$ & $\mathrm{N}$ & 30 \\
\hline & & & & & & & & $(-6,6)$ & W & 200 \\
\hline \multirow[t]{2}{*}{ AFGL 4029} & $2005 / 12 / 21$ & 1203 & 1.3 & 80 & 03:01:31.3 & $+60: 29: 12.9$ & 9 & $(0,0)$ & IRS1 & 70 \\
\hline & & & & & & & & $(24,1)$ & IRS2 & 70 \\
\hline \multirow[t]{2}{*}{ AFGL 961} & $2003 / 11 / 12$ & 1204 & 1.0 & 620 & $06: 34: 37.7$ & $+04: 12: 44.4$ & 10 & $(0,0)$ & $\mathrm{E}$ & 250 \\
\hline & & & & & & & & $(-5,-2)$ & W & 60 \\
\hline \multirow[t]{4}{*}{ W3 } & $2005 / 12 / 21$ & 501,100 & 1.4 & & $02: 25: 41.4$ & $+62: 06: 21.8$ & 11 & $(0,0)$ & IRS5 & 1300 \\
\hline & & & & & & & & $(3,-7)$ & IRS6 & 70 \\
\hline & & & & & & & & $(-1,-12)$ & IRS7 & 160 \\
\hline & & & & & & & & $(-2,11)$ & MIRS1 & 15 \\
\hline Cep A & $2004 / 07 / 13$ & 602 & 1.4 & 70 & $22: 56: 18.0$ & $+62: 01: 49.5$ & 12 & $(0,0)$ & & 440 \\
\hline
\end{tabular}

(1) Hackwell et al. (1982); (2) Simon et al. (1984); (3) Kastner et al. (1992); (4) Tamura et al. (1991); (5) Thompson et al. (1998); (6) Longmore et al. (2006); (7) Sridharan et al. (2005); (8) Wynn-Williams et al. (1981); (9) Zapata et al. (2001); (10) Castelaz et al. (1985); (11) van der Tak (2005); (12) comparison with Spitzer images, see Fig. 29.

Table 3. Characteristics of two $24.5 \mu \mathrm{m}$ filters. $\Delta \lambda$ is measured from the $50 \%$ transmission cut-on to cut-off wavelengths and $\lambda_{0}$ is the half-way point between the two. Measurements made available by the manufacturer.

\begin{tabular}{lccc}
\hline \hline ID & $\begin{array}{c}\lambda_{0} \\
(\mu \mathrm{m})\end{array}$ & $\begin{array}{c}\Delta \lambda \\
(\mu \mathrm{m})\end{array}$ & $\begin{array}{c}\text { Peak transmission } \\
(\%)\end{array}$ \\
\hline Q24.5-OLD & 24.47 & 1.91 & 64 \\
Q24.5-NEW & 24.56 & 0.75 & 64 \\
\hline
\end{tabular}

whilst only a part of the array is read out with the old one since radiation from the sky quickly saturates the well.

Chop-subtracted frames were stacked using the IMALIGN task (with cubic-spline interpolation) in the $\operatorname{IRAF}^{1}$ data reduction package. Flux calibration was achieved against either Cohen

\footnotetext{
${ }^{1}$ IRAF is distributed by the National Optical Astronomy Observatories, which are operated by the Association of Universities for Research in Astronomy, Inc., under cooperative agreement with the National Science Foundation.
}

(Cohen et al. 1995, 1999) or Sloan (Sloan et al. 2003) standards. We selected stars at similar airmasses to the target MYSOs whenever possible; however, in some cases when this was not feasible, we scaled the standard flux to the airmass of the relevant object by the atmospheric extinction relationships measured on 2002/12/15 UT (0.57 mag per airmass) and on 2003/11/12 UT (0.56) for the $Q 24.5-O L D$ filter. Note that these extinction values would probably only apply to this specific filter, along particular lines of sight, and at the times of observations. In some other instances (e.g. 2005/12/20 UT), even this airmass correction was not possible due to lack of appropriate data, and we reluctantly accepted the airmass mismatch as additional uncertainty. We estimate the overall uncertainty in flux calibration to be of the order of $10 \%$. The largest contribution usually comes from the absolute calibration uncertainty in the standard flux templates. Details of the target observations and extracted fluxes are given in Table 2. Images are not astrometrically calibrated. Positional offsets of the various sources in our images are with respect to the brightest $24.5 \mu \mathrm{m}$ source, 


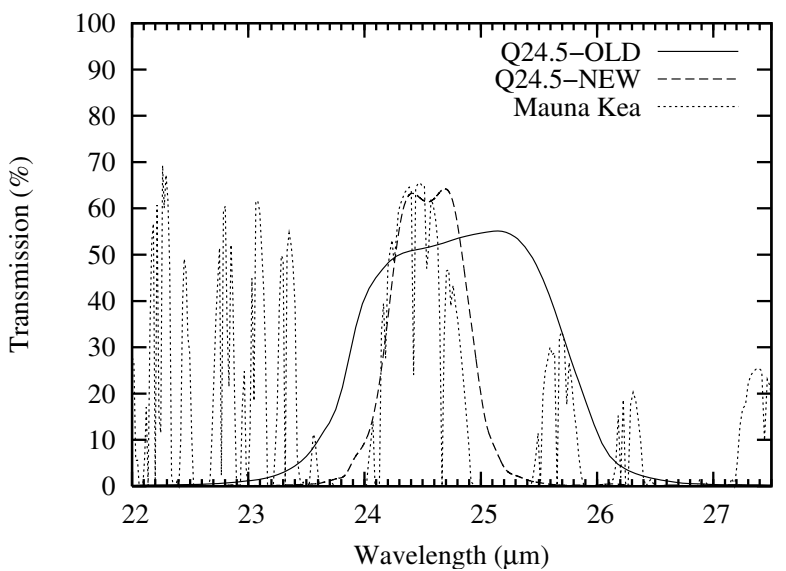

Fig. 1. Transmission curves for the $Q 24.5-O L D$ filter (solid line), $Q 24.5$ - NEW (dashed line), and the atmosphere above Mauna Kea (dotted line). The filter transmission spectra have been made available by the manufacturer. The atmospheric transmission curve has been calculated using USF HITRAN-PC for a standard "US tropical model" with an $\mathrm{H}_{2} \mathrm{O}$ partial pressure of $1.35 \mathrm{mmHg}$ at $4200 \mathrm{~m}$ looking through the atmosphere at a zenith angle of $30^{\circ}$ (Tomono 2000).

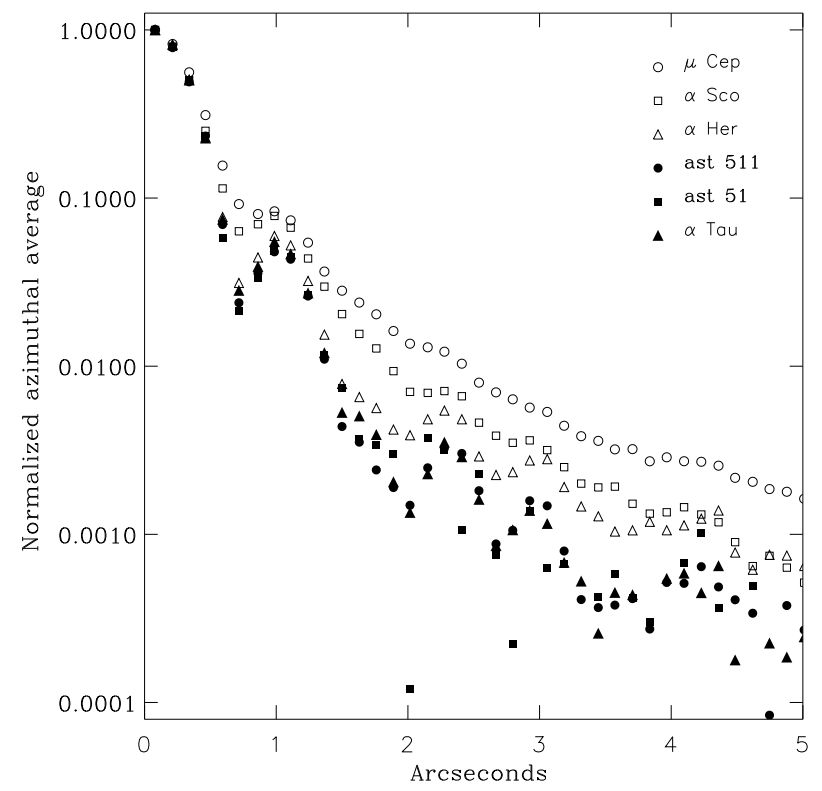

Fig. 2. Comparison of azimuthally averaged intensity profiles of the observed standard stars. Standard stars represented by open symbols are apparently surrounded by extended envelopes (see text).

generally identifiable with the brightest MYSO in each region presented in this paper.

\subsection{Point spread function reference stars}

We spend a few words on the point spread function (PSF) reference objects, as they are crucial in our analysis of comparing model images to the resolved MYSO emission (see Sect. 3.3). All standard star observations are given in Table 1. To test the validity of both the PSF standards and the flux standards as a model for the instrumental PSF, we investigate their azimuthally averaged intensity profiles in Fig. 2. The figure makes clear that three of the flux standard stars are actually extended, and therefore do not qualify as a PSF standard. They are the following objects:

$\mu$ Cep: is one of the largest stars in our Galactic neighbourhood. The star was observed a total of six times with COMICS. Detailed analysis shows this star to be extended in such degree and complexity, that we report on this star in a separate publication (de Wit et al. 2008). $\alpha$ Sco: is a supergiant star undergoing mass-loss. Mid-IR images at $12.5 \mu \mathrm{m}$ and at $20.8 \mu \mathrm{m}$ clearly show an extended distribution of circumstellar dust (Marsh et al. 2001).

$\alpha$ Her: is an irregular variable M star. Although the star mimics a PSF in the inner part of the profile (Fig. 2), its extent becomes apparent at radii $>2$ ". Interestingly, interferometric observations of the star have been interpreted as showing a mass loss event in the period 1989-1992. The material has an expansion speed of approximately $170 \mathrm{mas} \mathrm{yr}^{-1}$ (Tatebe et al. 2007), and it should have reached a distance of $2.5^{\prime \prime}$ from the star at the time of the observations. This distance is comparable to the distance where the excess emission becomes apparent.

As a consequence of the above discoveries, most observations do not have a concomitant PSF reference observation. In addition, the instrumental PSF shows a non-symmetric pattern due to the mirror support structure that rotates on the sky, making the interpretation of non-symmetric structure in the partially resolved sources taken at different times difficult. A comparison of the azimuthally averaged intensity profile of the six $\mu$ Cep observations taken over a period of more than 1 year shows however very few differences (see de Wit et al. 2008). This provides confidence in the stability of the (azimuthally averaged) COMICS PSF, and we therefore construct a single reference PSF from the remaining three genuine point sources ( $\alpha$ Tau, and asteroids 51 and 511), which we use throughout the remainder of this paper.

\section{Results}

\subsection{Brief description of the images}

The $24.5 \mu \mathrm{m}$ images reveal that all principal MYSO sources in the fourteen targeted massive star forming regions are resolved. The sources are generally discrete and have fairly simple, circular morphologies on the sky suggesting that the emission is dominated by the circumstellar envelope. We compare the observed azimuthally averaged intensity profiles of the MYSO envelopes to 1-D dust radiative transfer calculations. For each target, we attempt to reach consistency between the spatially resolved $24.5 \mu \mathrm{m}$ emission and the emission seen at other wavelengths as represented by the SED. In addition, we describe the $24.5 \mu \mathrm{m}$ emission morphology in relation to known star formation activity in the following subsection.

\subsection{Method of analysis}

Model SEDs and images are calculated using DUSTY, a code that solves the scaled 1-D dust radiative transfer problem (see Ivezic \& Elitzur 1997). We use a spherically symmetric dust distribution illuminated by a central, unresolved star. Numerical solutions are independent of the star's bolometric luminosity, and the SED and images need to be scaled before making a comparison to the observations. The bolometric luminosity is the prime stellar parameter that sets the inner dust sublimation radius and thus the size scale of the envelope; an increase causes the size of the emitting region to be larger (as $r_{\text {subl }} \propto \sqrt{L_{\mathrm{bol}}}$ ). As a result the intensity profile strongly depends on the $L_{\mathrm{bol}}$ assumed for the 
Table 4. Overview of the modelling results. The graphical representation are presented in the figures to each subsection. References of adopted distances and observed luminosities are given in Col. 4. Models \#1 in Col. 5 are the best-fitting models. Models \#2 and \#3 are the best-fitting models for radial density powerlaws that bracket the model \#1 powerlaw (except for AFGL 2591). Column 6 and onwards are model parameters (see Sect. 3.2). Column 15 gives the angular size of the inner dust free region.

\begin{tabular}{|c|c|c|c|c|c|c|c|c|c|c|c|c|c|}
\hline $\begin{array}{l}\text { MYSO } \\
\text { (1) } \\
\end{array}$ & $\begin{array}{c}\text { Distance } \\
(\mathrm{kpc}) \\
(2)\end{array}$ & $\begin{array}{c} \\
\left(L_{\odot}\right) \\
(3) \\
\end{array}$ & $\begin{array}{r}\text { Ref. } \\
\text { (4) } \\
\end{array}$ & $\begin{array}{r}\text { Mod. } \\
\# \\
(5) \\
\end{array}$ & $\begin{array}{c} \\
\left(L_{\odot}\right) \\
(6) \\
\end{array}$ & $\begin{array}{l}p \text { dust } \\
(7)(8)\end{array}$ & $\begin{array}{l}T_{\text {subl }} \\
(\mathrm{K}) \\
(9) \\
\end{array}$ & (10) & $\begin{array}{r}R_{\text {out }} / r_{\text {subl }} \\
(11)\end{array}$ & $\begin{array}{r}r_{\text {subl }} \\
(\mathrm{AU}) \\
(12) \\
\end{array}$ & $\begin{array}{r}n_{0} \\
\left(\mathrm{~cm}^{-3}\right) \\
(13) \\
\end{array}$ & $\begin{array}{r}M_{\mathrm{tot}} \\
\left(M_{\odot}\right) \\
(14) \\
\end{array}$ & $\begin{array}{r}\Theta \\
(\mathrm{mas}) \\
(15) \\
\end{array}$ \\
\hline \multirow[t]{3}{*}{ S140 IRS1 } & 0.91 & $2.0 \times 10^{4}$ & 1,15 & 1 & $1.3 \times 10^{4}$ & $1.0 \mathrm{ISM}$ & 1500 & 62 & 1250 & 32 & $2.8 \times 10^{7}$ & 32 & 69 \\
\hline & & & & 2 & $7.6 \times 10^{3}$ & 0.5 ISM2 & 1000 & 18 & 750 & 63 & $5.5 \times 10^{5}$ & 39 & 138 \\
\hline & & & & 3 & $1.2 \times 10^{4}$ & 1.5 ISM2 & 1000 & 104 & 1000 & 90 & $6.0 \times 10^{7}$ & 43 & 199 \\
\hline \multirow[t]{3}{*}{ M8E-IR } & 1.8 & $3.5 \times 10^{3}$ & 2 & 1 & $8.0 \times 10^{3}$ & 1.25 ISM & 1000 & 64 & 3000 & 70 & $2.7 \times 10^{7}$ & 300 & 78 \\
\hline & & & & & $1.1 \times 10^{4}$ & $1.0 \mathrm{ISM}$ & 1000 & 64 & 3000 & 81 & $1.0 \times 10^{7}$ & 1100 & 90 \\
\hline & & & & & $6.0 \times 10^{3}$ & $1.5 \mathrm{ISM}$ & 1000 & 64 & 5000 & 61 & $5.5 \times 10^{7}$ & 130 & 68 \\
\hline \multirow[t]{3}{*}{ AFGL 2136} & 2.0 & $7.0 \times 10^{4}$ & 3,16 & 1 & $2.5 \times 10^{4}$ & 1.0 ISM & 1000 & 70 & 1000 & 124 & $8.3 \times 10^{6}$ & 370 & 123 \\
\hline & & & & & $2.7 \times 10^{4}$ & 0.5 ISM & 1000 & 38 & 750 & 120 & $6.0 \times 10^{5}$ & 300 & 119 \\
\hline & & & & & $3.0 \times 10^{4}$ & 1.5 ISM & 1000 & 120 & 2000 & 139 & $4.5 \times 10^{7}$ & 330 & 139 \\
\hline \multirow[t]{3}{*}{ AFGL 2591} & 1.0 & $2.0 \times 10^{4}$ & 4 & 1 & $7.1 \times 10^{3}$ & 1.5 ISM & 1000 & 170 & 5000 & 67 & $1.3 \times 10^{8}$ & 430 & 134 \\
\hline & & & & & $4.0 \times 10^{3}$ & 1.0 ISM & 1000 & 90 & 2000 & 49 & $2.4 \times 10^{7}$ & 270 & 98 \\
\hline & & & & 3 & $1.4 \times 10^{4}$ & 1.0 ISM & 1500 & 82 & 3000 & 33 & $3.1 \times 10^{7}$ & 240 & 66 \\
\hline \multirow[t]{3}{*}{ NGC 2264 IRS1 } & 0.8 & $3.5 \times 10^{3}$ & 5,17 & 1 & $1.5 \times 10^{3}$ & 1.5 ISM & 1000 & 90 & 5000 & 31 & $1.5 \times 10^{8}$ & 480 & 77 \\
\hline & & & & 2 & $1.1 \times 10^{3}$ & 1.25 ISM & 1000 & 100 & 2000 & 27 & $1.2 \times 10^{8}$ & 330 & 66 \\
\hline & & & & 3 & $1.1 \times 10^{3}$ & 1.0 ISM & 1000 & 40 & 2000 & 26 & $2.1 \times 10^{7}$ & 320 & 64 \\
\hline \multirow[t]{3}{*}{ S255 IRS3 } & 2.5 & $2.6 \times 10^{4}$ & 6,18 & 1 & $2.4 \times 10^{4}$ & 1.25 ISM & 1000 & 70 & 5000 & 120 & $1.7 \times 10^{7}$ & 2300 & 96 \\
\hline & & & & & $2.7 \times 10^{4}$ & $1.0 \mathrm{ISM}$ & 1000 & 58 & 2000 & 127 & $6.0 \times 10^{6}$ & 1200 & 102 \\
\hline & & & & 3 & $3.5 \times 10^{4}$ & 1.5 ISM & 1000 & 176 & 5000 & 149 & $6.0 \times 10^{7}$ & 2200 & 119 \\
\hline \multirow[t]{3}{*}{ AFGL 5180} & 1.5 & $1.1 \times 10^{4}$ & 7 & 1 & $7.0 \times 10^{3}$ & $1.0 \mathrm{ISM}$ & 1000 & 200 & 1250 & 66 & $4.3 \times 10^{7}$ & 450 & 88 \\
\hline & & & & & $6.3 \times 10^{3}$ & 0.5 ISM & 1000 & 100 & 1250 & 59 & $2.5 \times 10^{6}$ & 530 & 79 \\
\hline & & & & & $4.2 \times 10^{3}$ & 0.0 ISM & 1000 & 100 & 1250 & 43 & $1.9 \times 10^{5}$ & 460 & 57 \\
\hline \multirow[t]{3}{*}{ IRAS 20126} & 1.7 & $1.3 \times 10^{4}$ & 8,19 & 1 & $9.2 \times 10^{3}$ & 0.0 ISMz2 & 1000 & 116 & 1000 & 51 & $2.3 \times 10^{5}$ & 470 & 60 \\
\hline & & & & & $7.9 \times 10^{3}$ & 0.5 ISMz2 & 1000 & 130 & 1250 & 53 & $3.7 \times 10^{6}$ & 560 & 61 \\
\hline & & & & & $7.9 \times 10^{3}$ & 1.0 ISM2 & 1000 & 184 & 1250 & 73 & $3.6 \times 10^{7}$ & 50 & 85 \\
\hline \multirow[t]{3}{*}{ Mon R2 IRS3 } & 0.83 & $6.5 \times 10^{3}$ & 9,20 & 1 & $1.3 \times 10^{4}$ & 1.0 ISM & 1500 & 80 & 1000 & 31 & $3.8 \times 10^{7}$ & 26 & 74 \\
\hline & & & & & $8.0 \times 10^{3}$ & 0.5 ISM2 & 1000 & 16 & 750 & 64 & $4.6 \times 10^{5}$ & 36 & 154 \\
\hline & & & & 3 & $1.3 \times 10^{4}$ & 1.5 ISM2 & 1000 & 128 & 750 & 93 & $6.9 \times 10^{7}$ & 37 & 223 \\
\hline \multirow[t]{3}{*}{ AFGL 437S } & 2.7 & $2.4 \times 10^{4}$ & 10,21 & & $4.2 \times 10^{3}$ & 0.0 ISM & 1000 & 62 & 1000 & 42 & $1.5 \times 10^{5}$ & 180 & 31 \\
\hline & & & & & $6.5 \times 10^{3}$ & 0.5 ISM & 1000 & 8 & 750 & 60 & $2.6 \times 10^{6}$ & 160 & 44 \\
\hline & & & & & $6.9 \times 10^{3}$ & 1.0 ISM & 1000 & 198 & 750 & 65 & $4.6 \times 10^{7}$ & 170 & 48 \\
\hline \multirow[t]{3}{*}{ AFGL 4029} & 2.2 & $2.0 \times 10^{4}$ & 11,22 & 1 & $1.1 \times 10^{4}$ & $0.0 \mathrm{ISMz}$ & 1500 & 82 & 1250 & 17 & $4.0 \times 10^{5}$ & 57 & 15 \\
\hline & & & & & & $0.5 \mathrm{IS}$ & 1000 & 8 & 1250 & 54 & $10^{6}$ & 385 & 49 \\
\hline & & & & & & & 1000 & 130 & 3000 & 63 & $2.6 \times 10^{7}$ & 1355 & 57 \\
\hline \multirow[t]{3}{*}{ AFGL 961E } & 1.4 & $6.0 \times 10^{3}$ & 12,23 & & $5.5 \times 10^{3}$ & $0.5 \mathrm{ISMz}$ & 1000 & 32 & 750 & 38 & $1.6 \times 10^{6}$ & 25 & 54 \\
\hline & & & & & $5.6 \times 10^{3}$ & 0.0 ISM2 & 1500 & 28 & 750 & 18 & & 8 & 26 \\
\hline & & & & & $10^{3}$ & $1.0 \mathrm{ISMz}$ & 1000 & 48 & 3000 & 43 & $0^{7}$ & 239 & 62 \\
\hline \multirow[t]{3}{*}{ W3 IRS5 } & 1.8 & $1.9 \times 10^{5}$ & 13,24 & 1 & $7.0 \times 10^{4}$ & $1.0 \mathrm{ISM}$ & 1500 & 144 & 750 & 73 & $3.0 \times 10^{7}$ & 156 & 81 \\
\hline & & & & & $8.8 \times 10^{4}$ & 0.5 ISM & 1500 & 106 & 750 & 78 & $2.6 \times 10^{6}$ & 359 & 87 \\
\hline & & & & 3 & $6.3 \times 10^{4}$ & 1.5 ISM2 & 1000 & 196 & 750 & 208 & $5.0 \times 10^{7}$ & 284 & 231 \\
\hline Cep A & 8 & $2.5 \times 10^{4}$ & 14,25 & & & & & & & & & & \\
\hline
\end{tabular}

(1) Crampton \& Fisher (1974); (2) Simon et al. (1985); (3) Kastner et al. (1992); (4) van der Tak et al. (1999); (5) Walker (1956); (6) Moffat et al. (1979); (7) Snell et al. 1988; (8) Cesaroni et al. (2005); (9) Herbst \& Racine (1976); (10) Alvarez et al. (2004); (11) Becker \& Fenkart (1971); (12) Cohen et al. (1973); (13) Imai et al. (2000); (14) Evans et al. (1981); (15) Lester et al. (1986); (16) Kastner et al. (1994); (17) Harvey et al. (1977); (18) Jaffe et al. (1984); (19) Cesaroni et al. (1997); (20) Thronson et al. (1980); (21) Wynn-Williams (1982); (22) Beichman et al. (1979); (23) Harvey et al. (1977); (24) Ladd et al. (1993); (25) Evans et al. (1981).

model. In practice, we determine the model $L_{\text {bol }}$ by minimising the difference between the model and observed SED. The model $L_{\text {bol }}$ can differ significantly from the observed $L_{\text {bol }}$, but for each model fit our adopted procedure finds the closest match between the two. In some case this match is poor and results in a large difference in observed and model $L_{\mathrm{bol}}$. Observed $L_{\mathrm{bol}}$ are listed for each target in Col. 3 of Table 4.

Other important model parameters are the outer radius of the envelope $\left(R_{\text {out }}\right)$ and the total amount of dust. The latter is parametrised in DUSTY by the total optical depth $A_{V}$. Higher values for these two parameters tend to increase the long wavelength flux levels. Of course, neither the total amount of dust nor the envelope's outer radius can have any arbitrary value. We constrain the extinction by the $9.7 \mu \mathrm{m}$ silicate absorption feature that is generally found to be strongly in absorption among MYSOs. The required data were in most cases provided by mid-IR spectra taken with the short wavelength spectrometer (SWS, de Graauw et al. 1996) on board the ISO satellite (Kessler et al. 1996). The ISO data have been obtained from ESA's ISO data archive ${ }^{2}$.

\footnotetext{
$\overline{2}$ Accessible at this URL http://iso.esac.esa.int/ida/
} 
The MYSO SEDs are constructed form the measured COMICS fluxes (Table 2) and from literature data. For most targets continuum measurements in the IR and (sub)mm are taken from the catalogue compiled by Gezari et al. (1999, available through Vizier), and includes IRAS and MSX observations. These data were supplemented, where possible, with more recent observations, especially the compilation by Mueller et al. (2002). The continuum slope of the ISO-SWS spectrum longwards of the silicate absorption is used to complete the SED at mid-IR wavelengths. In three cases Spitzer MIPS data are available. We extracted the photometry applying the non-linearity correction recipe by Dale et al. (2007). Data taken with large beams $\left(\gtrsim 15^{\prime \prime}\right)$ were generally avoided in the model fitting procedure. The following subsections, that are devoted to each object, highlight the used and discarded data in the fitting process. In the accompanying figures for each source open symbols are used to indicate large beam data not taken into account in the model fitting procedure, whereas filled symbols indicate the data that were actually used in the fitting procedure. Fluxes at wavelengths larger than $1.3 \mathrm{~mm}$ are excluded as they might be contaminated by freefree emission from ionized winds (e.g. Gibb \& Hoare 2007). Overall, the constructed SEDs cover a wavelength range from about $1 \mu \mathrm{m}$ to $1 \mathrm{~mm}$.

The model and observed images are compared in the fitting procedure by means of the azimuthally averaged intensity profile, normalized to its peak intensity. Model images are first scaled to model $L_{\mathrm{bol}}$ and convolved with the instrumental PSF. The intensity profiles are determined by binning and averaging the pixel counts in radial distance bins of size $0.13^{\prime \prime}$. The centre of the profile is found by minimising the pixel scatter as function of radial distance. We indicate the range in pixel counts found at each radius bin by an errorbar in the presented intensity profiles.

In order to perform a systematic comparison between models and observations, we have generated a standard grid that consists of 120000 DUSTY models. The grid probes the envelope parameter space and ranges in the following way:

- five radial density profiles. The radial density profile of the dust is described by a powerlaw of the form $n(r) \propto n_{0} r^{-p}$. The five density profiles cover power indices from $p=0.0$ to $p=2.0$ in steps of 0.5 .

- four types of dust mixtures. The dust size distribution is MRN (Mathis et al. 1977), and remains untouched. We use DL (Draine \& Lee 1984) opacities for silicates and combine this with either DL opacities for graphites or amorphous carbon (Zubko et al. 1996). Opacities are combined in a standard ISM mixture of $53 \%$ silicate and $47 \%$ graphite or a "twice ISM" mixture, i.e. $67 \%$ silicate and $33 \%$ graphite. Different mixtures may accommodate the silicate absorption profile for given total dust mass. The dust mixtures used have an opacity at $1.3 \mathrm{~mm}$ of $\kappa$ between $0.3-0.4 \mathrm{~g}^{-1} \mathrm{~cm}^{2}$, similar to the values of other types of dust in the literature, e.g. Ossenkopf \& Henning (1994);

- two dust sublimation temperatures $\left(T_{\text {subl }}\right): 1000 \mathrm{~K}$ and $1500 \mathrm{~K}$. The value for this parameter is the only unscaled parameter in DUSTY. The value of $T_{\text {subl }}$ has a large effect on the location of the dust sublimation radius but only a small effect on the normalized intensity profile. This is mainly due to a similar temperature dependence through the cloud with a very steep initial drop followed by a gradual decline. The similar temperature stratification leads to a similar normalized intensity profile. The different dust sublimation temperatures produce a different balance between short and long wavelength flux in the SED;
- three scaled sizes for the envelope. The outer radius is scaled to the inner sublimation radius. The standard model grid contains models with sizes 750, 1000 and 1250 times the inner dust sublimation radius, which correspond to typical envelope sizes of about $0.1 \mathrm{pc}$.

For each combination of these four envelope structure parameters, DUSTY calculates the resulting SEDs and images for an increasing total dust mass contained in the envelope. The $A_{V}$ grid goes from 2 to 200 in steps of 2, resulting in a grand total of 120000 models. The envelope parameters adopted in this grid lead to typical temperatures found at the outer radius of $20 \mathrm{~K}$ for $T_{\text {subl }}=1000 \mathrm{~K}$ for and $30 \mathrm{~K}$ for $T_{\text {subl }}=1500 \mathrm{~K}$ with typical densities at $R_{\text {out }}$ of $10^{3-4} \mathrm{~cm}^{-3}$.

The fitting procedure tries to find a model that fits the intensity profile and SED simultaneously. The SED fit includes the silicate absorption feature, slope of the ISO-SWS spectra, the far-IR SED peak and the (sub)mm continuum data. We aim to match the flux levels of the integrated (sub)mm continuum data providing additional constraints on the model parameters. The ISO spectra were rebinned logarithmically in 35 wavelength bins between 2.5 and $43 \mu \mathrm{m}$ in order to reduce its weight with respect to the continuum data. The binned spectrum probes the silicate feature at $9.7 \mu \mathrm{m}$ and the change in continuum slope longwards of it. In short, the fit procedure initially estimates the model $L_{\mathrm{bol}}$ by matching the overall shape of the scaled model SED to the observed one. DUSTY's output images are then accordingly scaled and convolved with the instrumental PSF. A comparison of the model SED and intensity profile to the observed ones is made for all generated models. A simple tally is performed based on a goodness-of-fit criterion. We apply a least-squares criterion for the SED fit, and a weighted least-squares criterion for the intensity profile fit. The latter uses weights that are inversely proportional to the square of the range in normalized intensity at each radial distance bin. This range is represented by errorbars in the intensity profile plots for each MYSO target. The model that fits both sets of data best is the one with the highest average ranking in the two tallies. If no simultaneous satisfactory fits were obtained, we changed the emphasis of the procedure in order to get fits to intensity profile and the long wavelength range of the SED only, i.e. dropping the requirement of fitting the near-IR and mid-IR part of the SED. In some cases no satisfactory model was found within our standard grid, and we chose to explore the effects of changes in envelope size. This is discussed in more detail in the subsections dedicated to each source.

Finally, the parameters for the underlying star are the same for each model and are the following. $L_{\mathrm{bol}}$ of the MYSOs are indicative of early B-type stars, thus we adopt a $T_{\text {eff }}$ of $25000 \mathrm{~K}$. There is only a negligible effect on the model dust emission for stars with slightly different effective temperatures. Distances for our sample stars were taken from the literature (see Table 4).

All final model parameters are listed in the modelling overview Table 4. For each MYSO we give the parameters for three models. Models \#1 correspond to the overall best-fitting model. Models \#2 and \#3 constitute the best-fit for models restricted to density powerlaws with indices that bracket the powerlaw index of the best-fitting model \#1, and are not the second and third best-fitting model. This is to illustrate how well the powerlaw index is constrained by the observables as this is our main priority; in some cases, models \#2 and \#3 do not provide acceptable fits. Therefore, the model parameters of Table 4 cannot be considered to represent the uncertainties on the model parameters. More detailed explanation can be found in the subsections dedicated to each MYSO. 


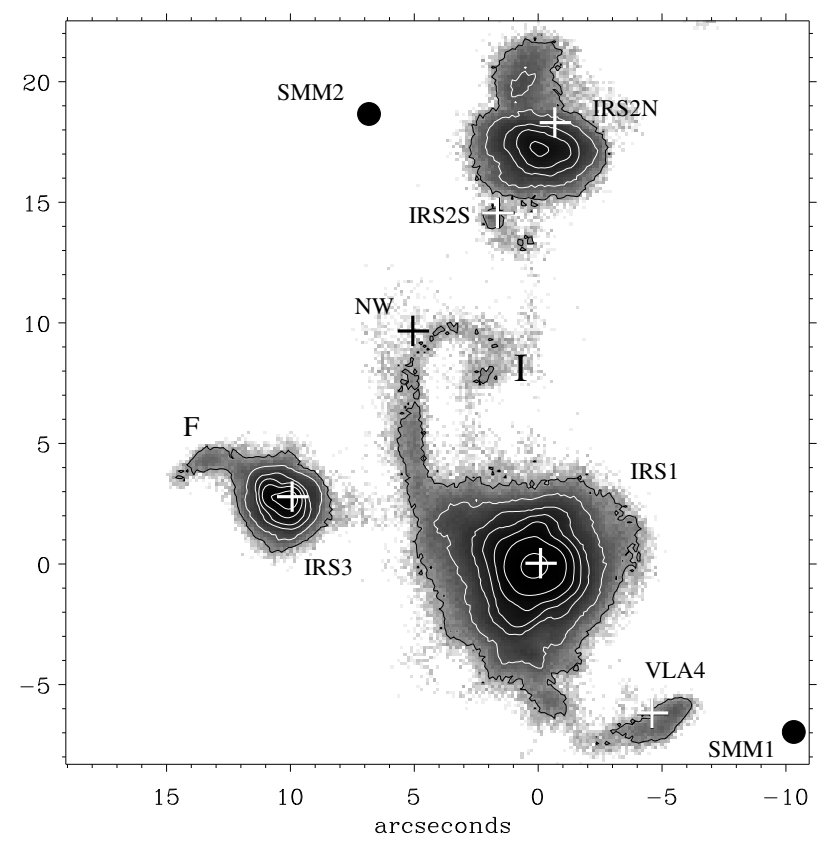

Fig. 3. COMICS $24.5 \mu \mathrm{m}$ image of the $\mathrm{S} 140$ region. The image is linearly scaled. Annotated objects are discussed in the text. Crosses correspond to radio sources (Evans et al. 1989; Tofani et al. 1995). Contour levels are at $0.5 \%, 1 \%, 2 \%, 3 \%, 5 \%, 10 \%$, and $40 \%$ of peak flux density $\left(5.9 \times 10^{2} \mathrm{Jy} \operatorname{arcsec}^{-2}\right)$. North is up, east is to the left.

\section{3. $24.5 \mu \mathrm{m}$ morphology and modelling}

\subsubsection{S140 (Figs. 3 and 4)}

Description: at a distance of 910 pc, S140 is a bright-rimmed cloud that forms the interface between an H II region and the molecular cloud L1204 (Crampton \& Fisher 1974). S140 contains an IR cluster of at least three sources (Beichmann et al. 1979). The main CO outflow direction in the region goes southeast north-west and a monopolar reflection nebula emanates from IRS1 which is associated with the blue-shifted south-east lobe (Hayashi et al. 1987; see also Hoare \& Franco 2007). The region displays spectacular arc-like features that are seen in high angular resolution $K$-band images (Forrest \& Shure 1986). They probably constitute material swept-up by outflow activity in the region (Weigelt et al. 2002). Recent cm continuum and $K$-band polarimetric imaging corroborate to suggest the presence of a disc-like structure for IRS1 oriented in a north-east south-west direction, perpendicular to the main outflow (Hoare 2006; Jiang et al. 2008).

Mid-IR morphology: the COMICS image of the S140 region is presented in Fig. 3. It reveals a wealth of features associated with previously known objects, the positions of which are labelled in the figure. Discrete peaks in mid-IR emission are found at the positions of IRS1, IRS2, and IRS3. The bright emission within 34 " of IRS1 has a fairly symmetric profile on the sky. The bright emission region $17^{\prime \prime}$ to the north of IRS1 coincides closely, but not exactly with the position of IRS2N according to the positions given in Tofani et al. (1995). IRS2S is identified with a point-like mid-IR source just south of that. IRS3 is found to be a triple system (Preibisch et al. 2003) and our image partly resolves the secondary object (IRS3b) at a distance of $0.75^{\prime \prime}$ east of the primary source (IRS3a).

The COMICS image also shows faint structures of diffuse emission. An arc of mid-IR emission concurs with the $K$-band arc labelled "I" by Weigelt et al. (2002). The curved wisp at
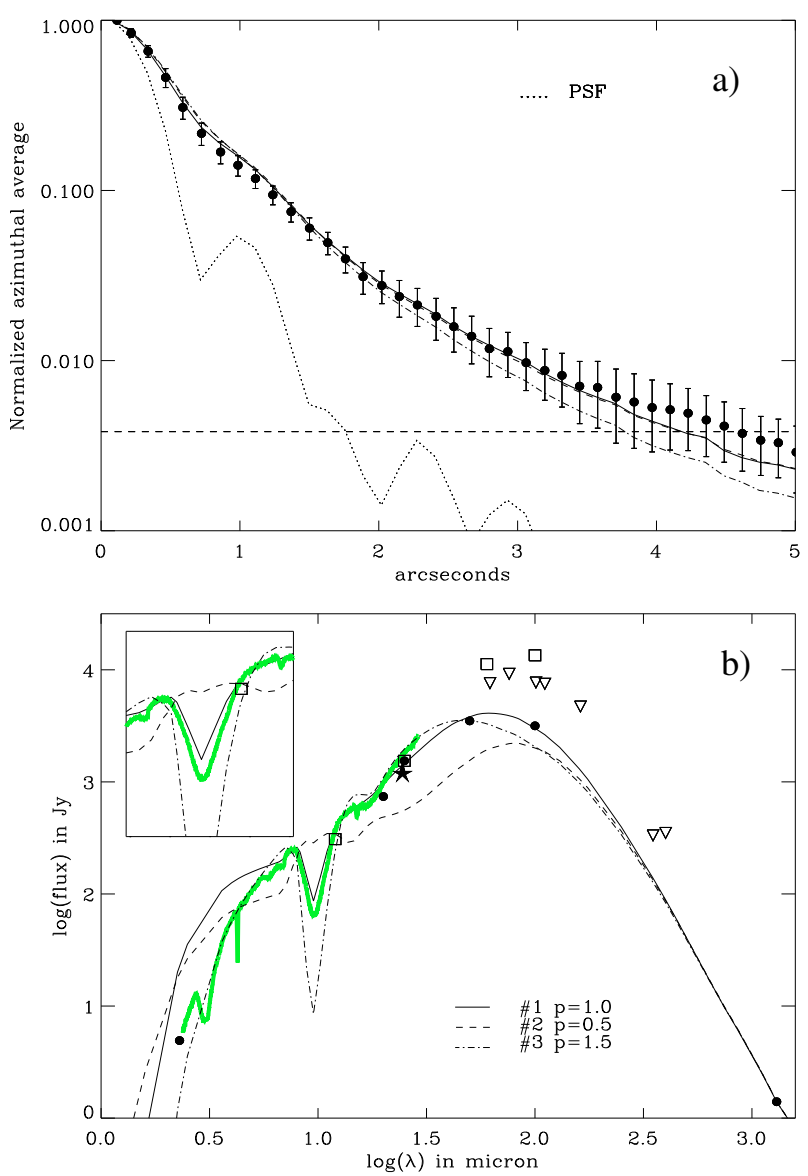

Fig. 4. Simultaneous model fits to the intensity profile and SED of S140 IRS1. Panel a) Observed (with errorbars) and three model normalized azimuthal intensity profiles at $24.5 \mu \mathrm{m}$. The code for the model line styles is the same as in panel b). The errorbars indicate the range in observed intensity values found at each radial distance bin. The horizontal dashed line equals three times the rms level of the normalized background level. Panel b) Observed (symbols) and model SEDs (various line styles). The COMICS flux measurement is represented by an asterisk. Open squares are IRAS measurements. Open triangles are large beam measurements discussed in the model results paragraph for each MYSO target. The thick line denotes the ISO-SWS spectrum. Models are fitted to the filled symbols and the logarithmically binned ISO-SWS spectrum only. The best fit model (model \#1 in Table 4) is indicated by the full line, models \#2, and \#3 by a dashed line, dot-dashed linestyle respectively. The best fitting model (\#1) has a $p=1.0$ radial density profile.

$\sim 3$ " from IRS3 corresponds to feature "F" of Preibisch et al. This feature is also known to have strongly polarised emission and strong $\mathrm{H}_{2}$ line emission, suggesting scattering and shocked material. Patches of diffuse emission are also found coincident with the radio sources VLA4 and NW (see Evans et al. 1989). This is the first time that the two radio sources are seen in the mid-IR. Previously (Evans et al. 1989), they were found coincident with the brightest parts of very extended near-IR nebulosity. Finally, we note that the two submm emission peaks (Minchin et al. 1995; Thompson et al. 2006) do not have $24.5 \mu \mathrm{m}$ emission counterparts. Clearly the image reveals that $24.5 \mu \mathrm{m}$ emission is not restricted to compact MYSO envelope emission. The emission has a faint diffuse character when it is associated with shocked dense material.

Model results: we focus the modelling on the main component in the region: bright MYSO IRS1. Its azimuthally averaged 


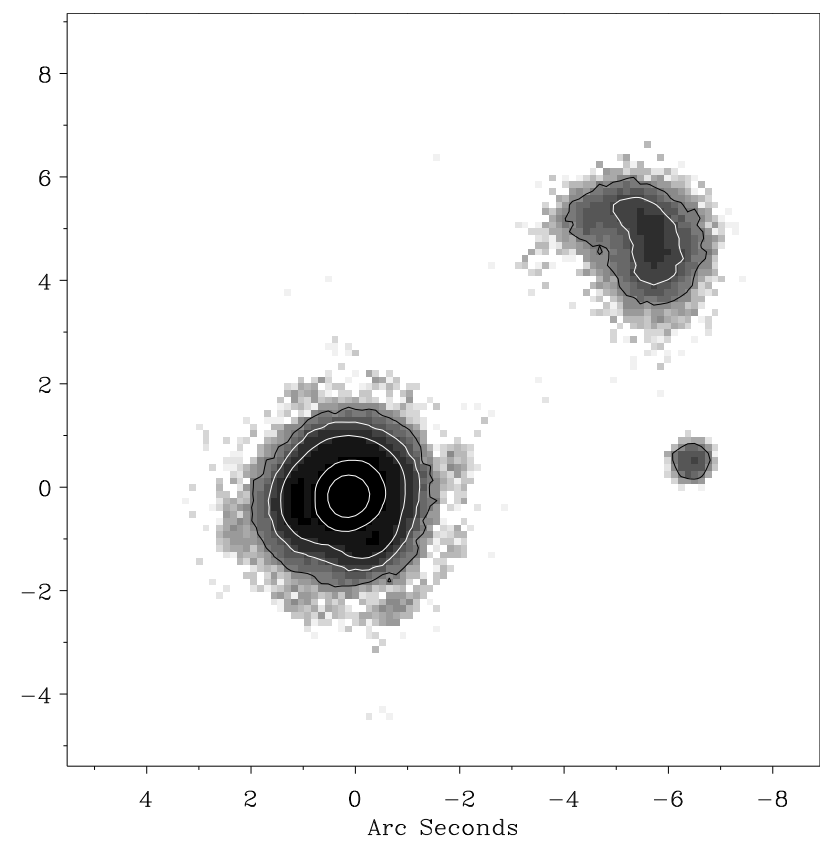

Fig. 5. COMICS $24.5 \mu \mathrm{m}$ image of the M8E region. Relative positions of the MYSO and the cometary shaped H II region are consistent with near-IR (2MASS) and radio images (Simon et al. 1984). Contour levels are at $1 \%, 2 \%, 5 \%, 10 \%$, and $40 \%$ of peak flux density $\left(2.4 \times 10^{2} \mathrm{Jy}_{\operatorname{arcsec}}-2\right)$. North is up, east is to the left.

$24.5 \mu \mathrm{m}$ intensity profile is presented in panel a of Fig. 4. The errorbars cover the peak to peak range in pixel counts measured at each radial distance bin. Their small values indicate that IRS1 can be considered to be symmetric to first order. We build the IRS1 SED (Fig. 4) from literature data. Photometry obtained using image restoration techniques applied to KAO continuum observations at 50 and $100 \mu \mathrm{m}$ by Lester et al. (1986) are preferred over large beam data $\left(50^{\prime \prime}\right)$ presented by Schwartz et al. (1983) and IRAS. We adopt for IRS1 the IRAM measurement at $1.3 \mathrm{~mm}\left(11^{\prime \prime} H P B W\right)$ by Gürtler et al. (1991). Large beam farIR and submm data by Schwartz et al. (1983) and Gürtler et al. (1991) are indicated by triangles in Fig. 4.

The intensity profile and the SED can be reproduced simultaneously by models with a $p=1.0$ radial density distribution. It is clear from Fig. 4 that the intensity profile can also be fit by model \#2, and to a somewhat lesser extent, model \#3. However, the simultaneous fit to the SED excludes shallow $p=0.5$ (model \#2) and steep $p=1.5$ (model \#3) models as viable alternatives. This is especially evident from their poor reproduction of the observed silicate absorption profile and the SED peak. On larger scales, modelling of the SED longwards of $100 \mu \mathrm{m}$ by Van der Tak et al. (2000) resulted in a $p=1.5$ density distribution. Mueller et al. (2002) find an overall best-fit to the SED and $350 \mu \mathrm{m}$ intensity profile for spherical models with $p=1.25$.

\subsubsection{M8E (Figs. 5 and 6)}

Description: M8E consists of a bright IR source and an optically thin radio source $8^{\prime \prime}$ to its north-west (Simon et al. 1984). The region shows evidence of a bipolar $\mathrm{CO}$ outflow, but it is not clear which of the two sources powers it (Simon et al. 1984). The IR object is resolved on scales of milli arcseconds by lunar occultation observations at $3.8 \mu \mathrm{m}$ and $10 \mu \mathrm{m}$. It can be interpreted as consisting of two physically different components (Simon et al. 1985): a compact hot component and a broad cooler component.
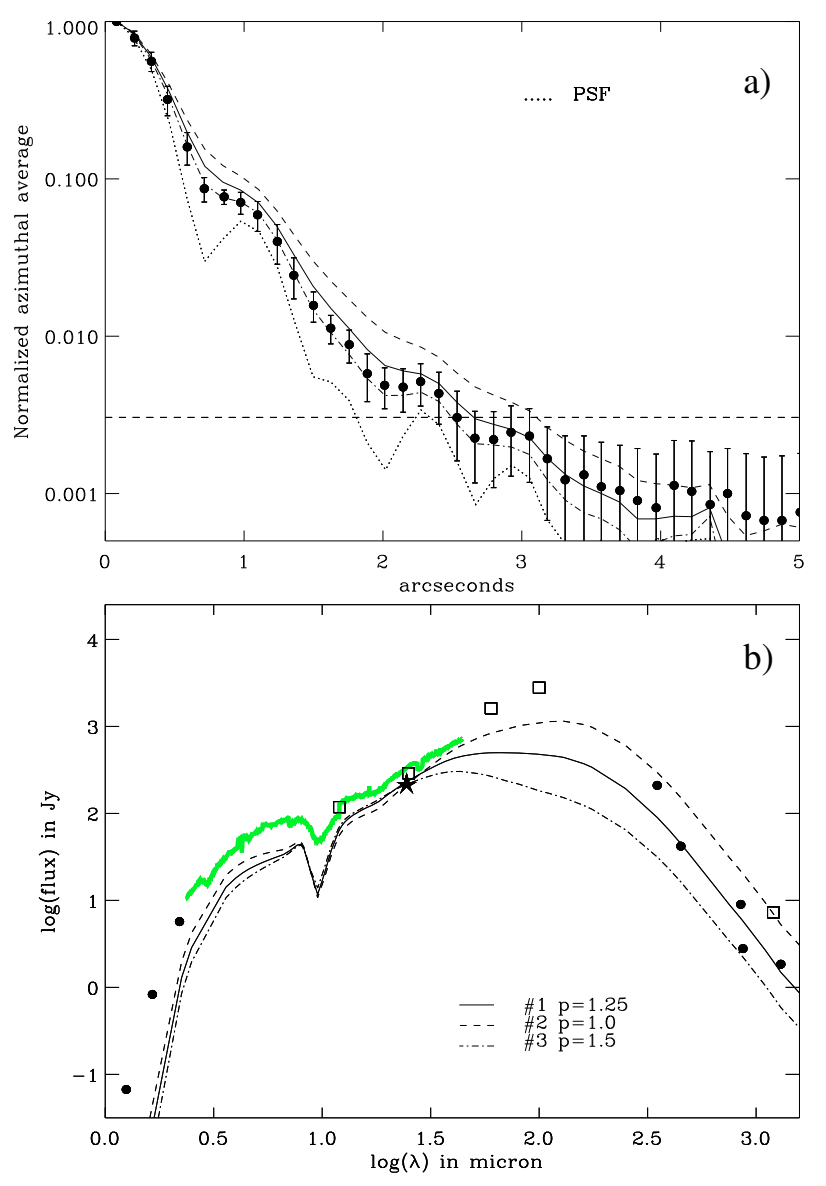

Fig. 6. As Fig. 4, but for M8E-IR. The SED data is described in Sect. 3.3.2. The best fitting model has $p=1.25$ radial density profile.

The broad component, that has an angular size of $0.1^{\prime \prime}$, does not correspond to the $30 \mathrm{~K}$ molecular cloud material causing the SED to peak at $100 \mu \mathrm{m}$, but is suggested to be a transition region between relatively hot disc material and cold cloud material. Mid-IR morphology: Fig. 5 shows the detection of M8E-IR as a compact discrete source which is symmetric to first order. The radio source emission is diffuse and has a cometary morphology. Its peak emission is found at a distance of $7.7^{\prime \prime}$ from M8E-IR. A third source is detected 6" west of M8E-IR.

Model results: the primary source of M8E-IR continuum data is from Mueller et al. (2002) and Gürtler et al. (1991). The six (sub)mm continuum measurements have been done with various beamsizes between $19^{\prime \prime}$ and $30^{\prime \prime}$, except the $870 \mu \mathrm{m}$ one that was performed with a $9^{\prime \prime}$ half-power beamwidth (Gürtler et al. 1991). For reference, we also plot the $1.2 \mathrm{~mm}$ datapoint obtained by Beltrán et al. (2006) with a half-power beamwidth of $26^{\prime \prime}$ (open square). It is clear that the intrinsic (sub)mm flux levels are uncertain, given the spread in flux levels in this wavelength range. At the short wavelength region we use the measurements by Simon et al. (1985).

M8E-IR is compact and only marginally resolved. In fact, the intensity profile is too steep for the bolometric luminosity derived from the observed SED. Steeper radial density powerlaws (creating a more compact cloud structure) lead to steeper intensity profiles, yet this advantage is offset by a decrease in flux at the long wavelength end. A commensurate increase in bolometric luminosity is required to fit the (sub)mm, but this in turn makes the intensity profile too shallow. Choosing a smaller 




Fig. 7. COMICS $24.5 \mu \mathrm{m}$ image of the AFGL 2136 region. The large crosses correspond to the peak emission of the three near-IR scattering nebulae (Kastner et al. 1994). Contour levels are at 2\%,5\%,10\%, and $40 \%$ of peak flux density $\left(1.1 \times 10^{2} \mathrm{Jy} \mathrm{arcsec}^{-2}\right)$. North is up, east is to the left.

outer radius is marred with the same problem, as is increasing the dust sublimation temperature.

The model that fits the intensity profile best has a $p=1.5$ powerlaw. Density profiles as shallow as $p=1.0$ are incompatible with the intensity profile. In order to increase the (sub)mm flux levels (without increasing the bolometric luminosity) we make the cloud larger than the sizes assumed in our model grid. Depending on the intrinsic (sub)mm flux levels, $p=1.25$ to $p=1.5$ are preferred with an outer radius of 3000 times the dust sublimation radius, or $\sim 1 \mathrm{pc}$. The flux levels of the ISO-SWS spectrum cannot be reproduced by any model SED. On scales of $1000-10000 \mathrm{AUs}$, the $350 \mu \mathrm{m}$ intensity profile is best represented by models with $p=1.75$ radial density distributions (Mueller et al. 2002), i.e. too steep to reproduce the $24.5 \mu \mathrm{m}$ intensity profile.

\subsubsection{AFGL 2136 (Figs. 7 and 8)}

Description: a three-lobed near-IR reflection nebula littered with some 30 faint point sources is illuminated by the dominant source IRS1. Near-IR polarisation indicates that IRS1 is illuminating conical cavities within a dusty envelope (Kastner et al. 1992). IRS1 is the driving source of an arcminute-scale bipolar CO outflow with a PA of $135^{\circ}$ (Kastner et al. 1994). Weak, optically thick radio emission originating from IRS1 is detected by Menten \& van der Tak (2004). The radio source is somewhat elongated in the south-easterly direction.

Mid-IR morphology: the COMICS image in Fig. 7 reveals one dominant source, IRS1, that is resolved and symmetric. The image is not very deep, and the envelope can be traced out to $\sim 3^{\prime \prime}$, where noise starts to dominate. IRS1 displays an extended wing to the south-east coincident with the near-IR "south" lobe of the nebula. A faint counter wing to the north-west would correspond to the "west" lobe. There are also traces of a $24.5 \mu \mathrm{m}$ counterpart to the faint "east" lobe (terminology from Kastner et al. 1992).
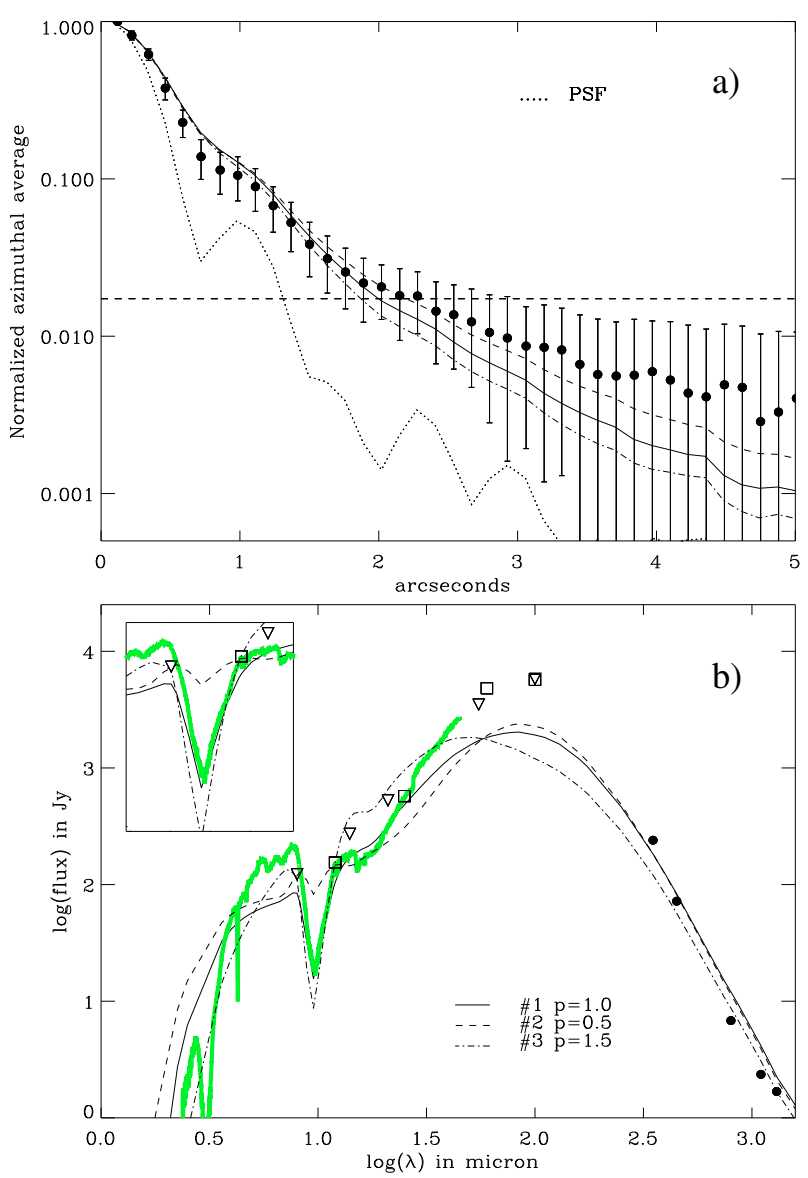

Fig. 8. As Fig. 4, but for AFGL 2136. The SED data is described in Sect. 3.3.3. The best fitting model has $p=1.0$ radial density profile.

The bright south lobe extends at roughly the same PA as the outflow activity.

Model results: the SED is built using the set of (sub)mm data from Kastner et al. (1994) obtained with the JCMT $\left(16.8^{\prime \prime}-18.5^{\prime \prime} H P B W\right)$, except the datapoint at $350 \mu \mathrm{m}$ which is from Mueller et al. (2002). IRAS measurements agree closely with the Harvey et al. (2000) data at 50 and $100 \mu \mathrm{m}$.

The intensity profile and SED of AFGL 2136 are best fit by a $p=1.0$ model. None of the models that can reproduce the intensity profile do a particular good job in reproducing the SED's short wavelength range, i.e. $<10 \mu \mathrm{m}$. At larger spatial scales, Mueller et al. (2002) and van der Tak et al. (2000) prefer steeper models with $p=1.75$ and $p=1.25$, respectively. Harvey et al. (2000) find reasonable fits to the 50 and $100 \mu \mathrm{m}$ intensity profiles and the SED, by dividing a $p=1.5$ model envelope in a high and low optical depth "hemisphere". We also find that certain $p=1.5$ models are capable of nicely fitting the full SED longwards of $5 \mu \mathrm{m}$ (not shown in Fig. 8), but they do not reproduce the $24.5 \mu \mathrm{m}$ intensity profile. The $p=1.5$ model shown in Fig. 8 does reproduce the intensity profile, but fits the SED worse than our preferred $p=1.0$ model.

\subsubsection{AFGL 2591 (Figs. 9 and 10)}

Description: various aspects of the AFGL 2591 envelope are discussed in van der Tak et al. (1999) using an extensive set of line and continuum observations. The authors conclude that the midIR and far-IR emission originates from the envelope, and that 


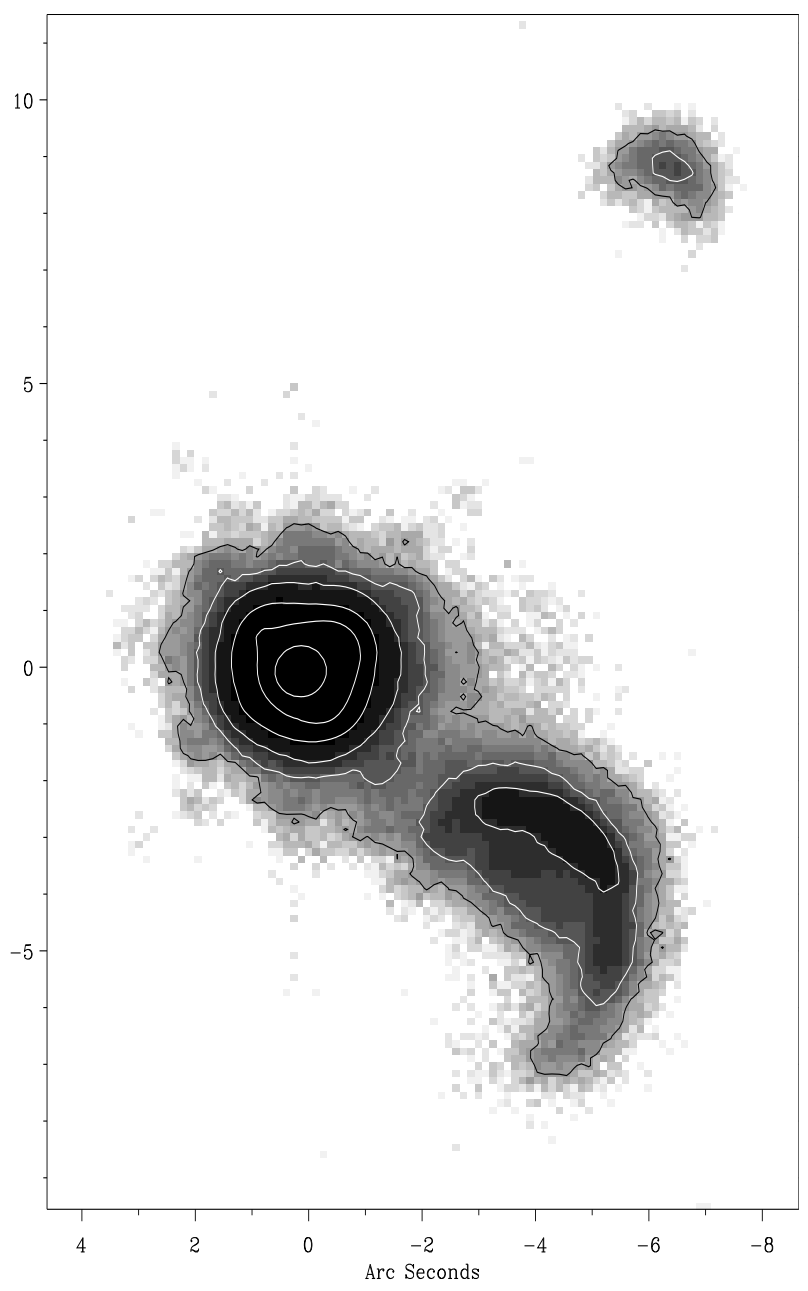

Fig. 9. COMICS $24.5 \mu \mathrm{m}$ image of the AFGL 2591 region. The relative positions of the MYSO and the two cometary shaped H II regions are consistent with the $8 \mathrm{GHz}$ sources in Tofani et al. (1995). Contour levels are at $0.5 \%, 1 \%, 2 \%, 5 \%, 10 \%$, and $40 \%$ of peak flux density $(7.7 \times$ $10^{2} \mathrm{Jy}_{\operatorname{arcsec}^{-2}}$ ). North is up, east is to the left.

the line-of-sight to the source nearly coincides with the opening cone carved by an east-west oriented bipolar $\mathrm{CO}$ outflow. There is ample evidence that the circumstellar material is not distributed in a spherically symmetric fashion and that a lowopacity pathway close to the line of sight has important effects on the source's appearance (Preibisch et al. 2003).

Mid-IR morphology: the COMICS image presents three sources: bright IRS1, a cometary shaped emission feature to the southwest, and a small emission region to the north-west. IRS1 is so bright that the spider diffraction pattern and the detector crosstalk (the vertical dark lane) are visible in Fig. 9. The cometary shaped feature to its south-west closely follows the optically thin H II region VLA1 (Wynn-Williams et al. 1977; Trinidad et al. 2003). The relative position of the source at the northern rim with respect to the other two is consistent with $8 \mathrm{GHz}$ source "n4" from Tofani et al. (1995).

Model results: in Fig. 10, we use JCMT submm data from Jenness et al. (1995), and CSO data from Mueller et al. (2002), that have comparable beamsizes (18" and $14^{\prime \prime}$, respectively). At mm wavelengths we show data from Gürtler et al. 1991) and one datapoint at $1.3 \mathrm{~mm}$ from Walker et al. (1990). The Gürtler et al. data were taken with a beam of $\sim 10^{\prime \prime}$ whereas the Walker et al. data have a $30^{\prime \prime}$ beam, possibly explaining the flux level
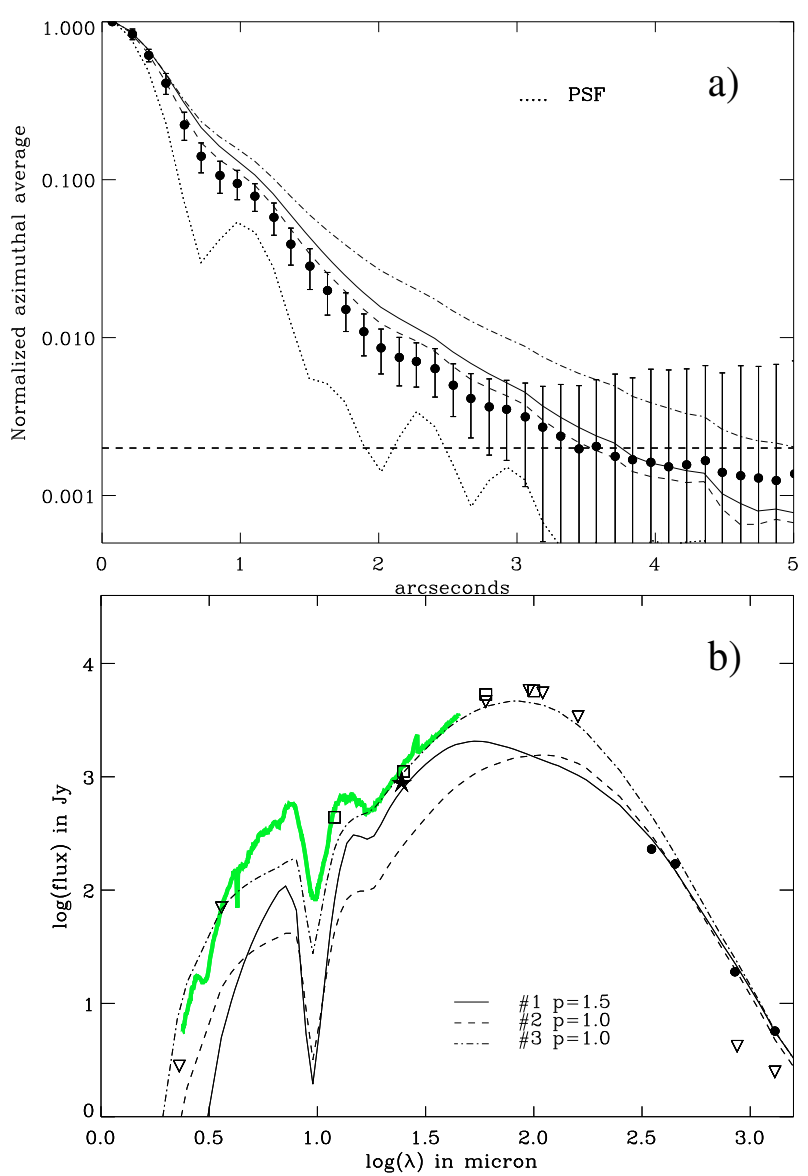

Fig. 10. As Fig. 4, but for AFGL 2591 IRS1. The SED data is described in Sect. 3.3.4. No simultaneous model fit to SED and intensity profile is found.

difference between these two sets. The far-IR KAO data of Lada et al. (1984) closely corresponds to the IRAS $60 \mu \mathrm{m}$ and $100 \mu \mathrm{m}$ photometry.

AFGL 2591 IRS1 presents an interesting case, as no simultaneous SED and intensity profile fit can be obtained. The bolometric luminosity implied by the observed SED impedes any reasonable model fit to the intensity profile (see e.g. the dotdashed $p=1.0$ model in Figs. 10a and b). We attempt to find a solution by simply fitting the (sub)mm SED and intensity profile. The compromise that does fit the $24.5 \mu \mathrm{m}$ flux and (nearly) the intensity profile is given by a $p=1.5$ model with a large outer radius of $\sim 5^{\prime}(1.6 \mathrm{pc})$. The discrepancy found between spherical models and AFGL 2591 is not limited to the mid and near-IR but is still severe at far-IR wavelengths. Such models require a substantial revision of the object's bolometric luminosity. In other words, the source is more compact than predicted by spherical models. A demonstration of a more appropriate 2-D modelling applied to this source is reported in Preibisch et al. (2003). These authors using speckle interferometry probing scales of $170 \mathrm{AU}$. They are able to model a resolved structure either as the inner rim of a circumstellar disc or as the dust sublimation radius of the MYSO envelope.

\subsubsection{NGC 2264 IRS1 (AFGL 989, Figs. 11 and 12)}

Description: a multi-wavelength study by Schreyer et al. (2003) suggests that NGC 2264 IRS1 is a young B-type star surrounded by low-mass companions located in a low-density cavity of a 


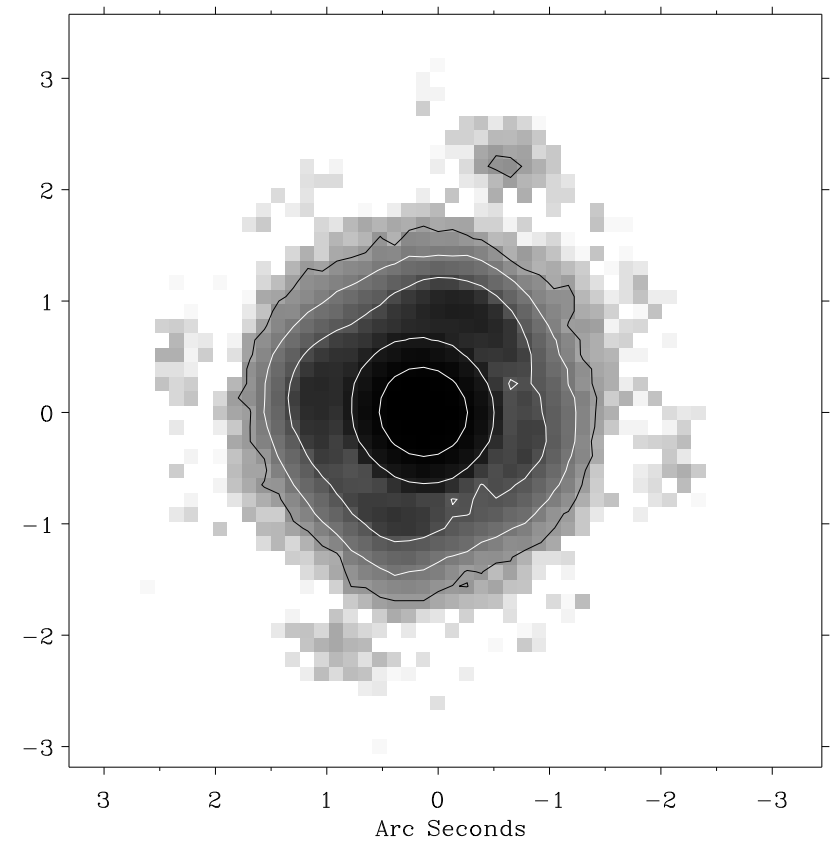

Fig. 11. COMICS $24.5 \mu \mathrm{m}$ image of NGC 2264 IRS1. Contour levels are at $1 \%, 2 \%, 5 \%, 10 \%$, and $40 \%$ of peak flux density $(4.1 \times$ $10^{2} \mathrm{Jy} \operatorname{arcsec}^{-2}$ ). North is up, east is to the left.

clumpy, shell-like, and dense cloud remnant. NGC 2264 IRS1 shows evidence for a $\mathrm{CO}$ outflow oriented along our line of sight (Schreyer et al. 1997). Attempts to resolve sub-arcsecond structure related to the CO outflow cavities in the near-IR with HST (Thompson et al. 1998) and using speckle techniques (Alvarez et al. 2004) failed. A reflection nebula on scales of arcseconds is clearly visible in the optical (Scarrott \& Warren-Smith 1989) and near-IR (Schreyer et al. 1997).

Mid-IR morphology: the COMICS image shows a single, symmetric object (IRS1). Most of the substructure seen in the image are diffraction patterns.

Model results: the model fits to SED and intensity profile are presented in Fig. 12. IRS1 coincides with submm source MMS5 (Ward-Thompson et al. 2000; Peretto et al. 2006) if one takes the correct near-IR coordinates (from 2MASS: $\alpha=06^{\mathrm{h}} 41^{\mathrm{m}} 10.16^{\mathrm{s}}$ and $\delta=+09^{\circ} 29^{\prime} 33.7^{\prime \prime}$ ) and the phase-referenced map by Nakano et al. (2003). We emphasise that the often reported lack of (sub)mm emission of IRS1 is due to wrong coordinates for IRS1 combined with poor astrometry (Ward-Thompson et al. 2000; Schreyer et al. 2003; Nakano et al. 2003; Peretto et al. 2006). Far-IR ( $25 \mu \mathrm{m}$ and onwards) observations presented in the literature are done with beams larger than 20" (Harvey et al. 1977; Chini et al. 1986; IRAS) and are considered to be upper limits. The model fit uses the JCMT and IRAM (sub)mm data presented in Ward-Thompson et al. (2000) with beam FWHM of $6^{\prime \prime}, 8^{\prime \prime}, 13^{\prime \prime}$ and $12^{\prime \prime}$ for the $350,450,800$ and $1300 \mu \mathrm{m}$ data, respectively. Archive Spitzer $70 \mu \mathrm{m}$ MIPS data are available for IRS1. We extracted the photometry applying the non-linearity correction recipe of Dale et al. (2007). A $70 \mu \mathrm{m}$ flux value of 960 Jy for IRS1 is used in the model fits.

The modelling indicates that bolometric luminosities that generate reasonable model fits to the intensity profile, find a rough correspondence with the mid-IR flux levels only if the powerlaw exponent equals $p=1.5$. The $70 \mu \mathrm{m}$ MIPS is not attained by any model. It is clear that any spherical model aimed at fitting the far-IR or (sub)mm part of the SED would need much
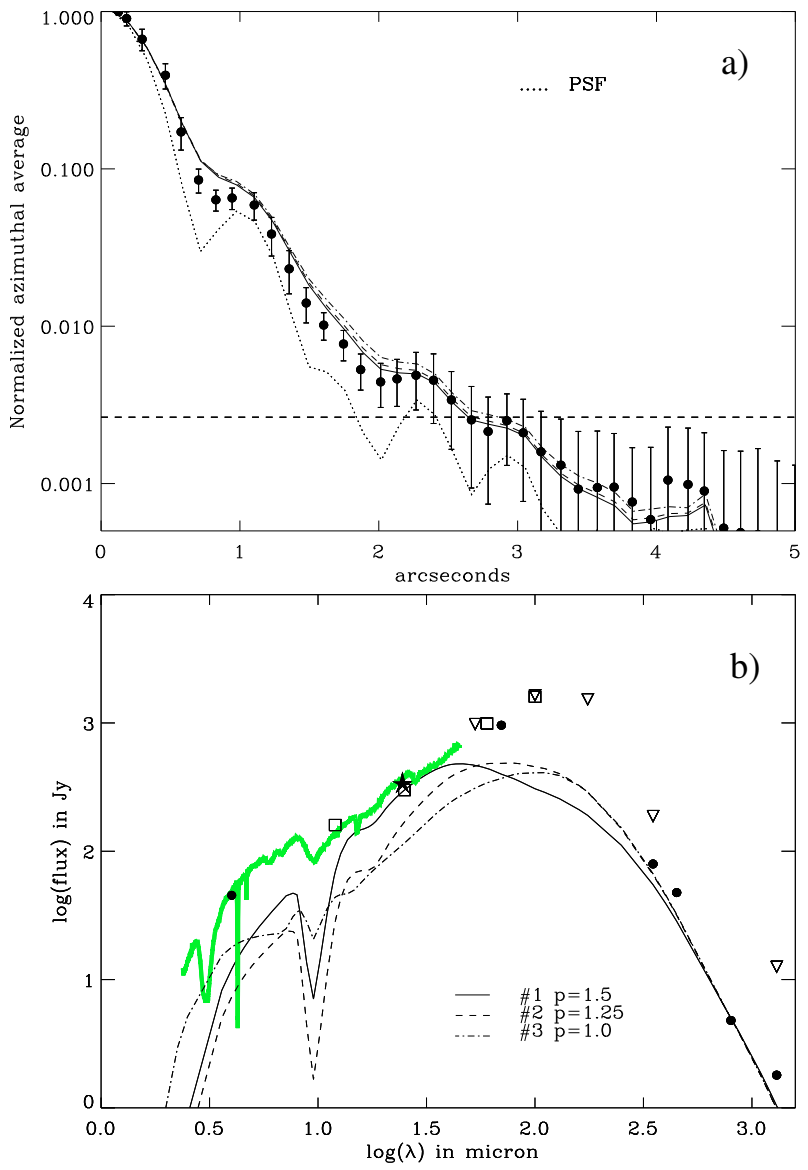

Fig. 12. As Fig. 4, but for NGC 2264 IRS1. The SED data is described in Sect. 3.3.5. The best fitting model has $p=1.5$ radial density profile.

larger luminosities, which is incompatible with the $24.5 \mu \mathrm{m}$ intensity profile.

\subsubsection{S255 (Figs. 13 and 14)}

Description: the region is dominated by two near-IR sources (NIRS1 and 3) and their bipolar IR reflection nebulae that were initially identified as the single source IRS1 (Beichman et al. 1979; Tamura et al. 1991; Itoh et al. 2001). High resolution nearIR speckle observations by Alvarez \& Hoare (2004) resolve the bipolar reflection nebula of NIRS1 (the western source), revealing it to be twisted in an "S" shape. High resolution Subaru nearIR polarimetric data indicate a depolarisation plane perpendicular to the near-IR bipolar nebula, suggesting the presence of a disc (Jiang et al. 2008).

Mid-IR morphology: the COMICS image shows NIRS3 to be the dominant source at $24.5 \mu \mathrm{m}$. Both mid-IR sources are found to be symmetric and resolved.

Model results: we focus our analysis on the bright source NIRS3 (the eastern source) for the SED and intensity profile fit. The spectral range of the ISO spectrum does not include the $9.7 \mu \mathrm{m}$ silicate feature. Instead we use the spectrum published in Willner et al. (1982), that describes the depth of the feature, but does not constrain the slope of the continuum longwards of it. The short wavelength range of the Willner et al. spectrum corresponds well with photometry taken by Evans et al. (1977). The SED uses (sub)mm data from the combined "core-envelope" 


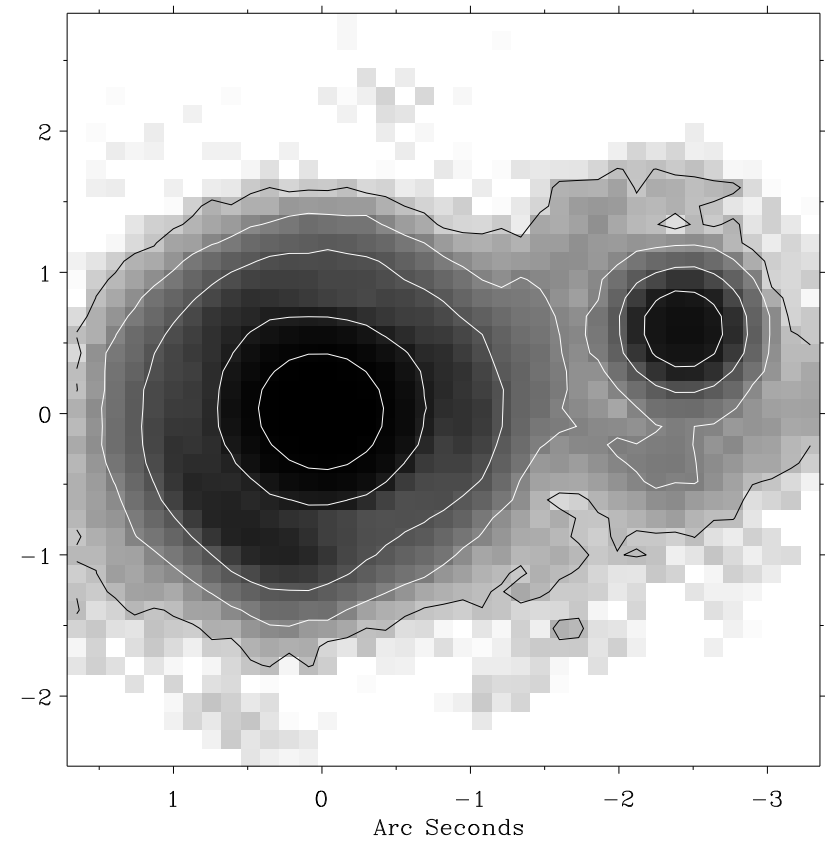

Fig. 13. COMICS $24.5 \mu \mathrm{m}$ image of the S255 region. Contour levels are at $1 \%, 2 \%, 5 \%, 10 \%$, and $40 \%$ of peak flux density $(1.9 \times$ $10^{2} \mathrm{Jy} \operatorname{arcsec}^{-2}$ ). North is up, east is to the left.

emission at $350 \mu \mathrm{m}$ with a $F W H P$ of $30^{\prime \prime}$ from Metzger et al. (1988), and measurements ( FWHP of 40") by Richardson et al. (1985). The mm measurement is by Chini et al. (1986a) obtained with the IRTF with a 90" beamsize. Other sets of (sub)mm points with higher fluxes are available in the literature and are represented by open triangles (Richardson et al. 1985; Metzger et al. 1988; Klein et al. 2005). The total COMICS flux level (i.e. NIRS1 and 3 taken together) is within 5\% of the MSX flux. More emission structure is hidden in the IRAS beam (see e.g. Longmore et al. (2006), for larger scale $18.7 \mu \mathrm{m}$ images).

S255 IRS3 constitutes a source that is mildly resolved at $24.5 \mu \mathrm{m}$. The intensity profile for IRS3 has been obtained by excluding the region between $\mathrm{PA}-45^{\circ}$ and $-135^{\circ}$, where emission from IRS 1 dominates. In Fig. 14 the observations are compared to three spherical envelope models, the parameters of which are listed in Table 4. The model with a radial density distribution with power exponent $p=1.25$ best fits both the SED and intensity profile. The $p=1.5$ model is clearly excluded as it requires high optical depths not substantiated by the silicate absorption profile. The case of S255 IRS3 is similar to the one encountered for M8E-IR. The models are required to have large envelopes which increase the long wavelength flux. This can be achieved without changing the bolometric flux, keeping the intensity profile unchanged.

\subsubsection{AFGL 5180 (Figs. 15 and 16)}

Description: AFGL 5180 has a blueshifted CO flow with a PA of $\sim 130^{\circ}$ (Snell et al. 1986). Saito et al. (2006) show that the IRAS point source breaks up into two main (sub)mm cores (see also Minier et al. 2005). One of these cores is centred on the near-IR source NIRS1 as identified by Tamura et al. (1991). The region reveals an intricate collection of $\mathrm{mm}$ cores mixed in with various near-IR sources (Tamura et al. 1991; Saito et al. 2007).

Mid-IR morphology: at $24.5 \mu \mathrm{m}$ and at the resolution of Subaru, AFGL 5180 consists of three sources: one extended source and two point-like sources. We follow the nomenclature by


Fig. 14. As Fig. 4, but for S255 IRS3. The SED data is described in Sect. 3.3.6. The best fitting model has $p=1.25$ radial density profile.

Tamura et al. (1991), in which the main discrete source can be identified as NIRS1 and the diffuse source as NIRS2. The region was recently imaged by Longmore et al. (2006) in the midIR. Their deconvolved images at $7.9 \mu \mathrm{m}$ shows that NIRS1 itself consists of three components that have a mutual distance of about $1 "$. The COMICS image shows that NIRS1 is somewhat asymmetric, but it is not resolved in multiple sources.

Model results: we concentrate our discussion on NIRS1, the main mid-IR source. Model comparisons to the observations are less well constrained than in the previous cases, because NIRS1 lacks a $10 \mu \mathrm{m}$ spectrum. An independent measure of the total optical depth is therefore absent. We use the (sub)mm photometry from Gear et al. (1988) and Thompson et al. (2006). The Gear et al. data have FWHM beamsizes of about an arcminute, whereas the Thompson et al. data are taken with SCUBA with $F W H M$ beamsizes of $8^{\prime \prime}$ and $14^{\prime \prime}$ for the $450 \mu \mathrm{m}$ and $850 \mu \mathrm{m}$ observations. The far-IR datapoint is from Ghosh et al. (2000). For the mid-IR we use the MSX photometry, that have a good correspondence with the IRAS data points. We find reasonable fits to the intensity profile for $p=0.0, p=0.5$ and $p=1.0$ radial density distributions (see Fig. 16), but the MSX and IRAS points in the SED are better reproduced by the $p=1.0$ model. Any steeper distributions may provide equally good fits, except that they would require optical depths exceeding $A_{\mathrm{V}}=200^{m}$ and much larger outer radii then adopted in our grid, which are typical for most sources in our sample. The $p=1.0$ model already requires a comparatively large optical depth with respect to the other MYSO envelopes for it to fit the MSX data. 




Fig. 15. COMICS $24.5 \mu \mathrm{m}$ image of the AFGL 5180 region. The principal western source corresponds to NIRS1 (Tamura et al. 1991). Contour levels are at $5 \%, 10 \%$, and $40 \%$ of peak flux density $(4.5 \times$ $10^{2} \mathrm{Jy} \operatorname{arcsec}^{-2}$ ). North is up, east is to the left.

\subsubsection{IRAS 20126+4104 (Figs. 17 and 18)}

Description: IRAS 20126 is one of the best examples of a relatively massive young star that is actively fed by an accretion disc. The star is thought to have a mass of approximately $7 M_{\odot}$ (Cesaroni et al. 2005). The system is known to show jet/outflow phenomena at a PA of about $-60^{\circ}$ (see Cesaroni et al. 2005; Su et al. 2007), with the jet oriented almost perpendicular to the line of sight (Cesaroni et al. 1999). At wavelengths shorter than $20 \mu \mathrm{m}$ a "dark lane" separates two emission regions (Sridharan et al. 2005; De Buizer 2007). This morphology could be either due to the presence of two tight clusters (in which case the dark lane does not correspond to a physical structure) or due to a silhouetted disc with emission emanating from the cavity walls of the outflow either side of the disc (De Buizer 2007).

Mid-IR morphology: the COMICS image consists of two emission regions. The morphology of the north-west source (source "5" in De Buizer 2007) corresponds to the one presented in Shepherd et al. (2000) at $17.9 \mu \mathrm{m}$ and coincides with the direction of the outflow. The south-eastern peak emission overlaps the two sources that are seen separated by a dark lane at shorter wavelengths. No separation due to a dark lane is detected, although it is extended perpendicular to the dark lane seen at shorter wavelengths. The two sources have a separation of $0.7^{\prime \prime}$ in $18.3 \mu \mathrm{m}$ images by De Buizer (2007). The somewhat lower resolution at 24.5 micron is probably partly responsible for the failure to separate the two sources. In addition, it seems likely that lower extinction and thermal emission from the putative dark lane itself could be blurring the distinction between the two emission components. It would argue in favour of the dark lane being a physical structure rather than a clearance of emitting material as suggested in De Buizer (2007).

Model results: the ISO-SWS spectrum has a low SNR at wavelengths shorter than $10 \mu \mathrm{m}$. This includes the silicate absorption profile, inhibiting a independent measure of the envelope's optical depth. A sharp rise of the spectrum towards longer wavelengths indicates a very high extinction. In the model fits we use the photometry collected by Hofner et al. (2007, and references
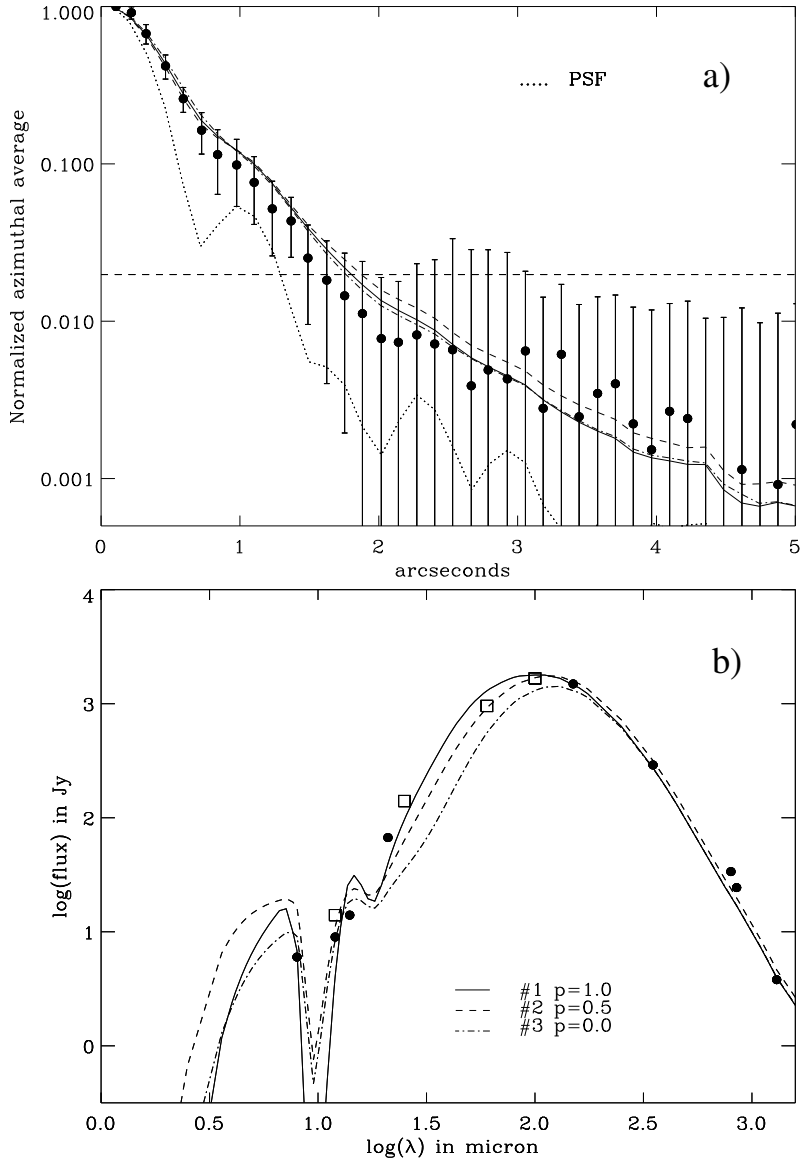

Fig. 16. As Fig. 4, but for AFGL 5180. The SED data is described in Sect. 3.3.7. The best fitting model has $p=1.0$ radial density profile.

therein). We fit only the large beam data presumably corresponding to the dusty halo. The submm observations are obtained with the JCMT by Cesaroni et al. (1999) with HPBW between 7" and $14^{\prime \prime}$. The intensity profile is obtained from the region that excludes emission from the north-western source. We find good simultaneous fits to both intensity profile and SED, with a preference for flat radial density distributions with powerlaw indices $p=0.0$ and $p=0.5$. Steeper density distributions are not able to fit the $24.5 \mu \mathrm{m}$ intensity profile. These values for the density distribution power index are at odds with Hofner et al. (2007) and van der Tak et al. (2000). The former find consistency between the observed SED and what one would expect from an accretion disc embedded in a spherical infalling halo (on scales of $10^{\prime \prime}$ ) with $p=1.5$. Van der Tak et al. (2000) find $p=1.75$, on scales larger than the ones probed by the $24.5 \mu \mathrm{m}$ image.

\subsubsection{Mon R2 (Figs. 19 and 20)}

Description: the central region of the Mon R2 cloud consists of at least five bright IR sources within $\sim 0.25 \mathrm{pc}$ (Beckwith et al. 1976). The region shows complex submm dust continuum emission which is well illustrated by the $850 \mu \mathrm{m}$ map by Giannakopoulou et al. (1997). High resolution near-IR imaging $\left(0.075^{\prime \prime}\right.$ resolution) resolves the brightest mid-IR source (IRS3) into a triple system surrounded by strong diffuse nebulosity (Preibisch et al. 2002).

Mid-IR morphology: the COMICS mosaic in Fig. 19 shows IRS3 to be the dominant discrete source at $24.5 \mu \mathrm{m}$. The large, 




Fig. 17. COMICS $24.5 \mu \mathrm{m}$ image of IRAS 20126+4104. Contour levels are at $5 \%, 10 \%, 20 \%, 40 \%$, and $70 \%$ of peak flux density $(4.7 \times$ $10^{1} \mathrm{Jy} \operatorname{arcsec}^{-2}$ ). North is up, east is to the left.

$30^{\prime \prime}$ scale mid-IR shell structure is identifiable in near-IR continuum emission in which case it is due to dust scattering (Howard et al. 1994). Its morphology corresponds roughly to the extent of the blister H II region (Massi et al. 1985). If we compare the midIR image with these maps, we may identify the $24.5 \mu \mathrm{m}$ emission with the walls of the ionized region. Maximum intensity along the mid-IR ridge corresponds to the location of the source IRS1. The image does not reveal any point source at this position. IRS2, IRS3 and IRS5 are all observed to be extended sources. IRS3 is elongated with a PA along the direction of the main binary (Preibisch et al. 2002).

Model results: Submm and mm imaging at $870 \mu \mathrm{m}$ and $1.3 \mathrm{~mm}$ (HPBW of $18^{\prime \prime}$ and 23", respectively) by Henning et al. (1992) resolve and identify the various components of the cloud with the IR sources. We show in Fig. 20 the intensity profile and SED fits to IRS3, the most luminous mid-IR source of the region. Figure 20b also shows the Mueller et al. (2002) compilation of continuum measurements at the long wavelengths, including the $350 \mu \mathrm{m}$ CSO measurement (HPBW 14"). The flux level of the ISO spectrum corresponds closely to the COMICS flux measurement. The intensity profile levels out at distances $>5^{\prime \prime}$ which is probably due to contribution in flux by the diffuse emission in the region.

The case of Mon R2 IRS3 is similar to S140 IRS1. Simultaneous model fit to the intensity profile and SED shows that models with a radial density profile of $p=1.0$ are preferred. Again steep radial profiles are excluded as they require high optical depths in order to provide a good fit to the intensity profile.

\subsubsection{AFGL 437S (Figs. 21 and 22)}

Description: AFGL 437 is a compact cluster of few dozen nearIR sources located at a distance of $2.7 \mathrm{kpc}$ (Wynn-Williams et al. 1981; Weintraub \& Kastner 1996). The cluster is dominated by four bright sources $437 \mathrm{~N}, \mathrm{~S}, \mathrm{E}$ and $\mathrm{W}$. The source $437 \mathrm{~W}$ dominates the radio emission, whereas only weak radio emission is measured towards the 437S. (Kurtz et al. 1994; Torrelles et al. 1992). Alvarez et al. (2004) resolve a monopolar sub-arcsecond



Fig. 18. As Fig. 4, but for IRAS 20126+4104. The SED data is described in Sect. 3.3.8. The best fitting models have $p=0.0$ or $p=0.5$ radial density profiles.

near-IR nebula from 437S. Water masers have been detected towards $437 \mathrm{~W}$ and $437 \mathrm{~N}$ (Torrelles et al. 1992). Weintraub \& Kastner (1996) find that $437 \mathrm{~N}$ actually breaks up into two components (see also Rayner \& McClean 1987). The south-eastern source of the two (named WK34) is the most embedded and found to be responsible for the region's molecular outflow and the dominant source of the near-IR reflection nebula (see also Meakin et al. 2005).

Mid-IR morphology: the COMICS image is dominated by the $\mathrm{UCH}$ II region associated with $437 \mathrm{~W}$. No discrete counterpart is found for $437 \mathrm{~W}$, only a diffuse emission region. The $437 \mathrm{~N}$ source is resolved into two components, with the driving source WK34 the most luminous of the two. Of the 4 sources detected, $437 \mathrm{~S}$ is the most luminous and marginally resolved by our observations at $24.5 \mu \mathrm{m}$.

Model results: we concentrate our analysis on 437S. Submm and mm observations were performed by Dent et al. (1998) with the JCMT at four wavelengths with beamsizes between $16^{\prime \prime}$ and $19^{\prime \prime}$. The ISO-SWS spectrum of the region is dominated by emission from the H II region and is discarded. Instead we use the $10 \mu \mathrm{m}$ photometry of $437 \mathrm{~S}$ taken by Wynn-Williams et al. (1981). The model fitting procedure discards the IRAS photometry, but takes into account the COMICS flux measurement.

The spatial information derived from the marginally resolved source is not enough to strongly constrain the various radial density distributions. We show in Fig. 22 three models that reproduce the intensity profile and the continuum emission in the SED. We find that $p=1.0$ profiles require large optical depth 


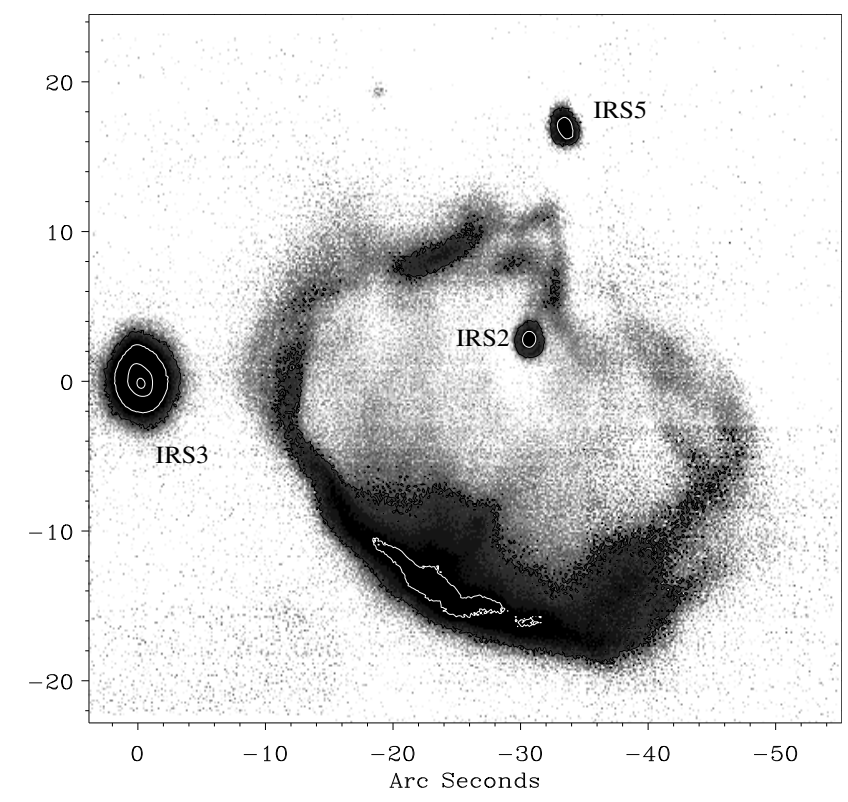

Fig. 19. COMICS $24.5 \mu \mathrm{m}$ image of the Mon R2 region. Contour levels are at $1 \%, 3 \%, 20 \%$, and $70 \%$ of peak flux density $(5.0 \times$ $10^{2} \mathrm{Jy} \operatorname{arcsec}^{-2}$ ). North is up, east is to the left.

in order to fit the (sub)mm and the $24.5 \mu \mathrm{m}$ data. This seems to be excluded by the silicate absorption profile. We therefore prefer rather shallow radial density distributions with $p=0.0$ or $p=0.5$, but with considerable uncertainty.

\subsection{Complex sources}

MYSO sources presented in this section show evidence for multiple condensations within $1^{\prime \prime}$ of the profile centre (AFGL 4029, AFGL 961, and W3 IRS5). Azimuthally averaged intensity profiles yield consequently large uncertainties, which constrain the models only little. In the case of the fourth source presented in this section, the Cep A star forming region, no discrete central source could be identified at all. We discuss the $24.5 \mu \mathrm{m}$ images alongside some literature background for each of these four objects.

\subsubsection{AFGL 4029 (Figs. 23 and 24)}

Description: Beichman et al. (1979) have shown that the region consists of two sources at 10 and $20 \mu \mathrm{m}$, separated by about $20^{\prime \prime}$. Both mid-IR sources have radio emission (Kurtz et al. 1994). IRS1 shows evidence for outflow activity. Zapata et al. (2001) find IRS1 to consist of a double radio source, interpreted to be a binary object. They identify the southern of the two sources responsible for the outflow activity.

Mid-IR morphology: the COMICS image reveals two emission regions. A compact emission region identified as IRS1 which is shown in Fig. 23, and a diffuse emission region associated with IRS2 located at 20" from IRS1 (not shown in Fig. 23). IRS1 shows a discrete source with evidence for at least two condensations, the southern being the brightest one. In addition at least two bands of emission are located within the inner 2 ", rendering IRS 1 a rather patchy source at $24.5 \mu \mathrm{m}$. Finally, IRS 1 has an extended wing $\left(\sim 4^{\prime \prime}\right)$ to the west. Scattered light emission and thermal emission due to extended nebulosity to the west of IRS1 is discussed in Deharveng et al. (1997) and Zavagno et al. (1999). The $24.5 \mu \mathrm{m}$ morphology of IRS1 follows closely the
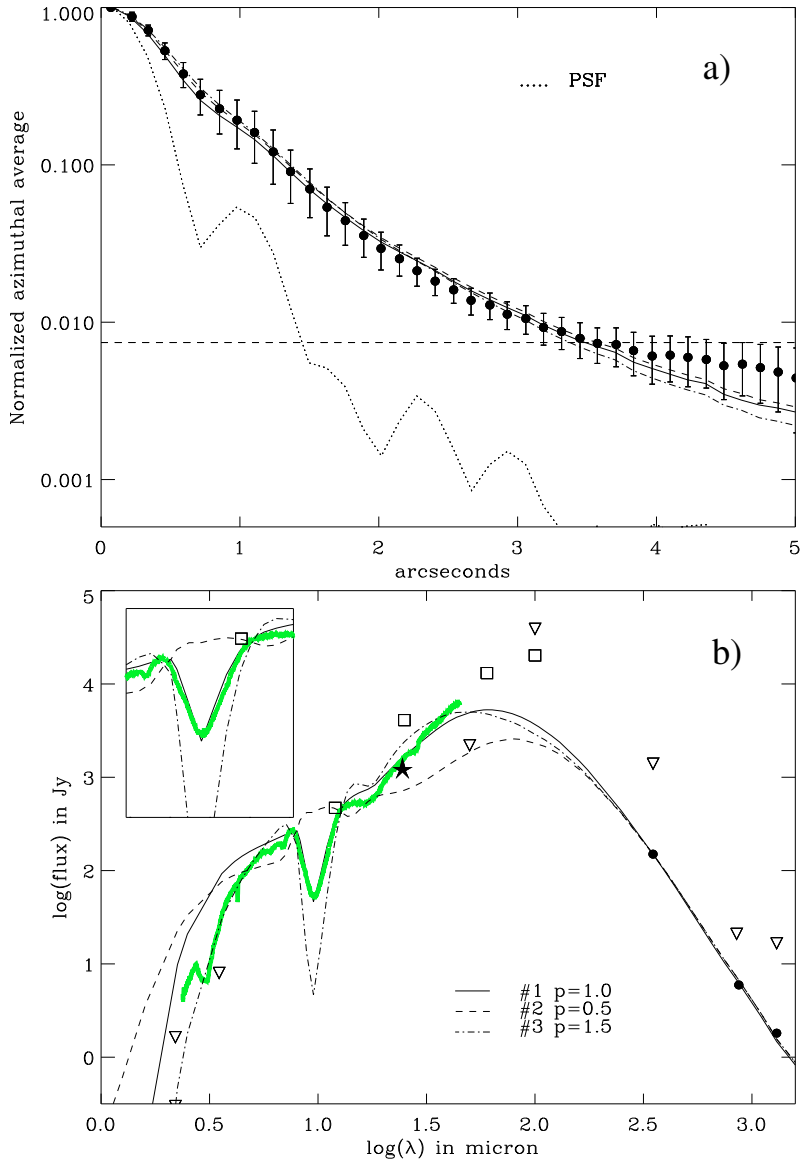

Fig. 20. As Fig. 4, but for Mon R2 IRS3. The SED data is described in Sect. 3.3.9. The best fitting model has $p=1.0$ radial density profile.

radio morphology as presented in Zapata et al. (2001). The midIR wing is possibly the counterpart to the east-west extension seen in high-resolution radio maps.

Model results: the mid-IR part of the SED is adopted from data presented in Zavagno et al. (1999). IRAM 1.27 mm flux (beamsize $\sim 15^{\prime \prime}$ ) is taken from Klein et al. (2005), and submm SCUBA fluxes (beamsizes of $8^{\prime \prime}$ and 14 " for the $450 \mu \mathrm{m}$ and $850 \mu \mathrm{m}$ ) are presented in Di Francesco et al. (2008). Finally a Spitzer MIPS flux measurement gives a flux density of $680 \mathrm{Jy}$ for this source.

AFGL 4029 presents an intensity profile that is characterized by a large scatter in intensity, as represented by the errorbars in Fig. 24, due to the presence of patchy emission within $2^{\prime \prime}$. The lack of a $10 \mu \mathrm{m}$ spectrum for the source also complicates the analysis. The data of Zavagno et al. (1999) cover the wings of the silicate absorption profile, providing some constraints. Although the intensity profile shows a large scatter, simultaneous model fits to SED and intensity profile are hard to find; the basic problem being the extent of the source. This translates into a very luminous object, incompatible with the SED. In Fig. 24, we show models with relatively flat density profiles $(p=0.0$ or $0.5)$, steeper profiles are incompatible with the intensity profile.

\subsubsection{AFGL 961 (Figs. 25 and 26)}

Description: the source is a well-known double object located in the outskirts of the Rosette Nebula (Lenzen et al. 1984) and surrounded by a stellar cluster (Aspin 1988). The east and west components have a separation of about $6^{\prime \prime}$ and display outflow 


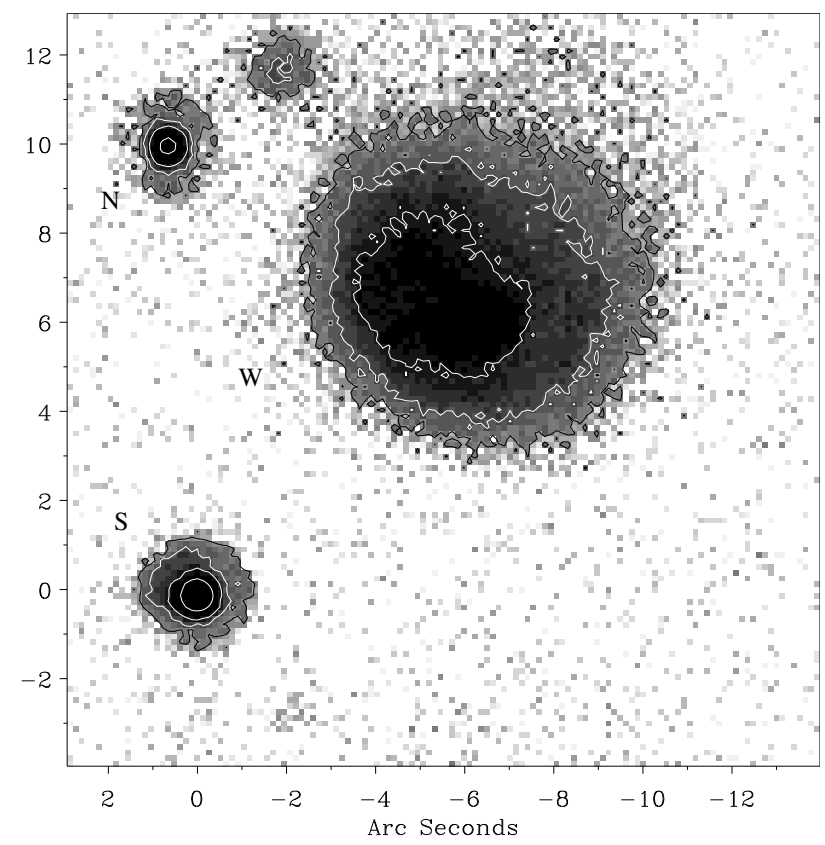

Fig. 21. COMICS $24.5 \mu \mathrm{m}$ image of the AFGL 437 region. Contour levels are at $5 \%, 10 \%, 20 \%$, and $50 \%$ of peak flux density $(3.2 \times$ $10^{1} \mathrm{Jy}_{\operatorname{arcsec}^{-2}}$ ). North is up, east is to the left.

phenomena (Lada \& Gautier 1982). Snell \& Bally (1986) determined the radio spectral index, finding it to be consistent with an ionized wind.

Mid-IR morphology: the dominant source in the COMICS image is AFGL 961E. The western source $961 \mathrm{~W}$ is discrete and somewhat extended in a north-westerly direction. AFGL 961E displays two diffuse blobs to the north-west and south-west of the peak emissions.

Model results: Chini et al. (1986) model this source and find good correspondence with a spherical model for wavelengths shorter than $100 \mu \mathrm{m}$. An emission excess with respect to their spherical model is found for wavelengths longer than this. We use SCUBA measurements at $450 \mu \mathrm{m}$ and $850 \mu \mathrm{m}$ (beamsizes of $8^{\prime \prime}$ and $14^{\prime \prime}$ ), which are significantly lower. Further, we use the spectrum taken by Willner et al. (1982), mid-IR photometry from Cohen (1973), Simon \& Dyck (1977), and the $350 \mu \mathrm{m}$ IRTF measurement $(H P B W$ 90") is from Gürtler et al. (1991). We measured the $70 \mu \mathrm{m}$ flux from archival Spitzer MIPS data, finding a flux of 720 Jy for the source. The broad SED and intensity profile are well fit by $p=0.5$ density powerlaws with a moderate optical depth. Steeper density profiles do not fit the intensity profile, and would additionally require either a higher optical depth or a much larger envelope outer radius in order to fit the submm points.

\subsubsection{W3 (Figs. 27 and 28)}

Description: W3 is an important and complex region containing objects in various stages of the formation process (WynnWilliams et al. 1972; Claussen et al. 1994). One prominent source in the region is IRS5, which consists of a double source ( 1 " separation) at infrared wavelengths (Howell et al. 1981; van der Tak et al. 2005). IRS5 was discovered to harbour at least seven very compact radio continuum sources within a radius of $0.03 \mathrm{pc}$ (Claussen et al. 1994). A number of these are likely to be shock excited clumps in the surrounding molecular material,



Fig. 22. As Fig. 4, but for AFGL 437S. The SED data is described in Sect. 3.3.10. The best fitting model has $p=0.0$ radial density profile.

driven by embedded OB stars (Wilson et al. 2003; van der Tak et al. 2005). Water masers in IRS5 have been shown to trace two outflows, both in roughly the NS direction (Imai et al. 2000), a direction similar to the overall $\mathrm{CO}$ outflow of the region.

Mid-IR morphology: the main $24.5 \mu \mathrm{m}$ component in W3 region is the bright source IRS5. The double source at its centre is resolved, the northern one is the brightest of the two sources. IRS5 shows prominent diffuse emission that surrounds the double source. It extends in both NS and EW direction. IRS6 is a resolved source with the peak emission somewhat offset to the west of centre. IRS7 is a diffuse, cometary shaped emission region. IRS3 is resolved in various $24.5 \mu \mathrm{m}$ patches of diffuse emission. An unidentified source is found $10^{\prime \prime}$ north of IRS5.

Model results: data for W3 IRS5 are from van der Tak et al. (2005), the compilation made by Campbell et al. (1995), and the $10 \mu \mathrm{m}$ spectrum presented in Willner et al. (1982). The (sub)mm data have been obtained with beamsizes between 15" and 19".

Shallow radial density models provide good fits to the SED, but fail to reproduce the intensity profile. Models with $p=1.5$ powerlaw produce to much mid-IR flux requiring a high optical depth, leading to a too deep silicate absorption feature. The best fitting models require a $p=1.0$ powerlaw.

\subsubsection{Cep A (Fig. 29)}

Description: Patel et al. (2005) reported a flattened HCN structure to be a circumstellar disc near the HW2 radio jet, the brightest radio source of the region (Hughes \& Wouterloot 1984). The 


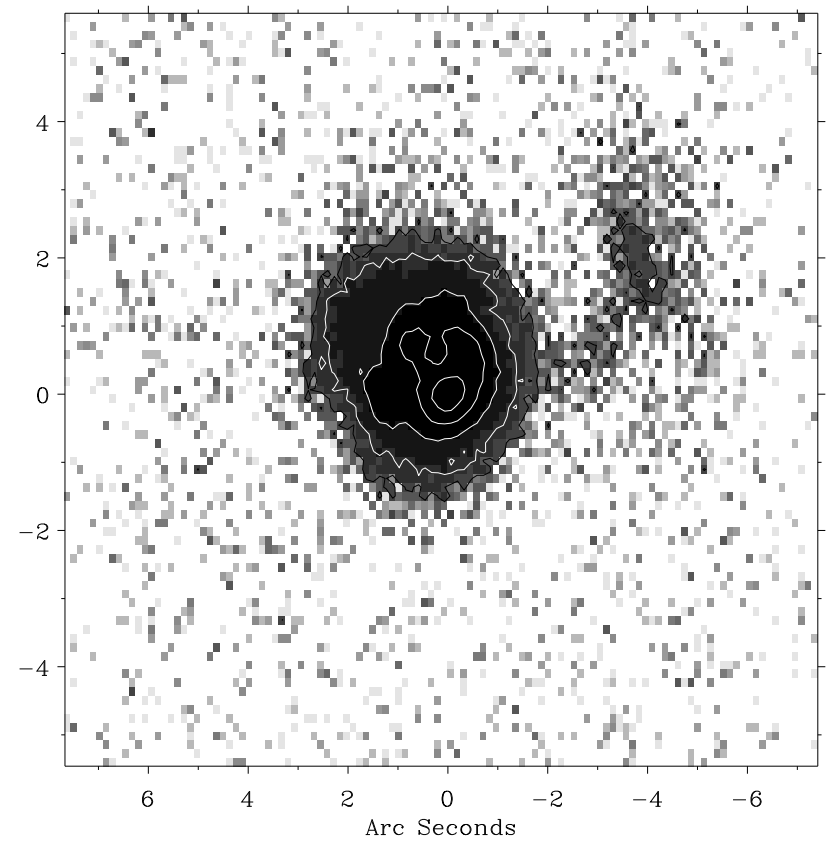

Fig. 23. COMICS $24.5 \mu \mathrm{m}$ image of the AFGL 4029 region. Contour levels are at $5 \%, 10 \%, 25 \%, 50 \%$, and $80 \%$ of peak flux density $(2.2 \times$ $10^{1} \mathrm{Jy} \operatorname{arcsec}^{-2}$ ). IRS2 is $20^{\prime \prime}$ to the east to IRS1, and not shown in the image. North is up, east is to the left.

radio jet is known to be the driving force of a large-scale molecular outflow in a north-east, south-west direction (Gómez et al. 1999). Jiménez-Serra et al. (2007) showed that the HCN disc is resolved into a much smaller disc and a hot core (Martín-Pintado et al. 2005). The centre of their disc falls close to the centre of the ionized HW2 jet as determined in Curiel et al. (2006).

Mid-IR morphology: the region shows a complex morphology comprising various arcs and patches. The bright $24.5 \mu \mathrm{m}$ emission corresponds to the bright near-IR reflection nebula that occupies the blue-shifted outflow cavity (Lenzen et al. 1984). The COMICS image does not show any point source and we performed a cross matching with a lower angular resolution, archival Spitzer $8 \mu \mathrm{m}$ image in order to determine a rough astrometric solution. The result shown in Fig. 29 is a reasonable match between 8 and $24.5 \mu \mathrm{m}$. Note that the $8 \mu \mathrm{m}$ emission corresponding to the $24.5 \mu \mathrm{m}$ emission peaks is saturated. HW2 appears to be hidden by high extinction just at the bottom tip of a dark lane.

Model results: Cep A is not modelled because direct radiation from the source is obscured at $24.5 \mu \mathrm{m}$.

\section{Summary of the model fitting}

We give a summary of the best-fitting models in Table 5 . Whether the best model reproduces certain important SED wavelength intervals and the $24.5 \mu \mathrm{m}$ intensity profile has been indicated by tickmarks and crosses. In some cases no such assessment could be made due to lack of data or data of insufficient quality. The table makes clear that spherical models are capable of reproducing at least part of the SED and intensity profile simultaneously. The models systematically fail to reproduce the short wavelength range, which is a well-known shortcoming of spherical geometries (e.g. Gürtler et al. 1991).

A pattern emerges from Table 5 in which the objects that are satisfactorily reproduced by spherical models (S140 IRS1, AFGL 2136, AFGL 5180, Mon R2 IRS3) are described by radial
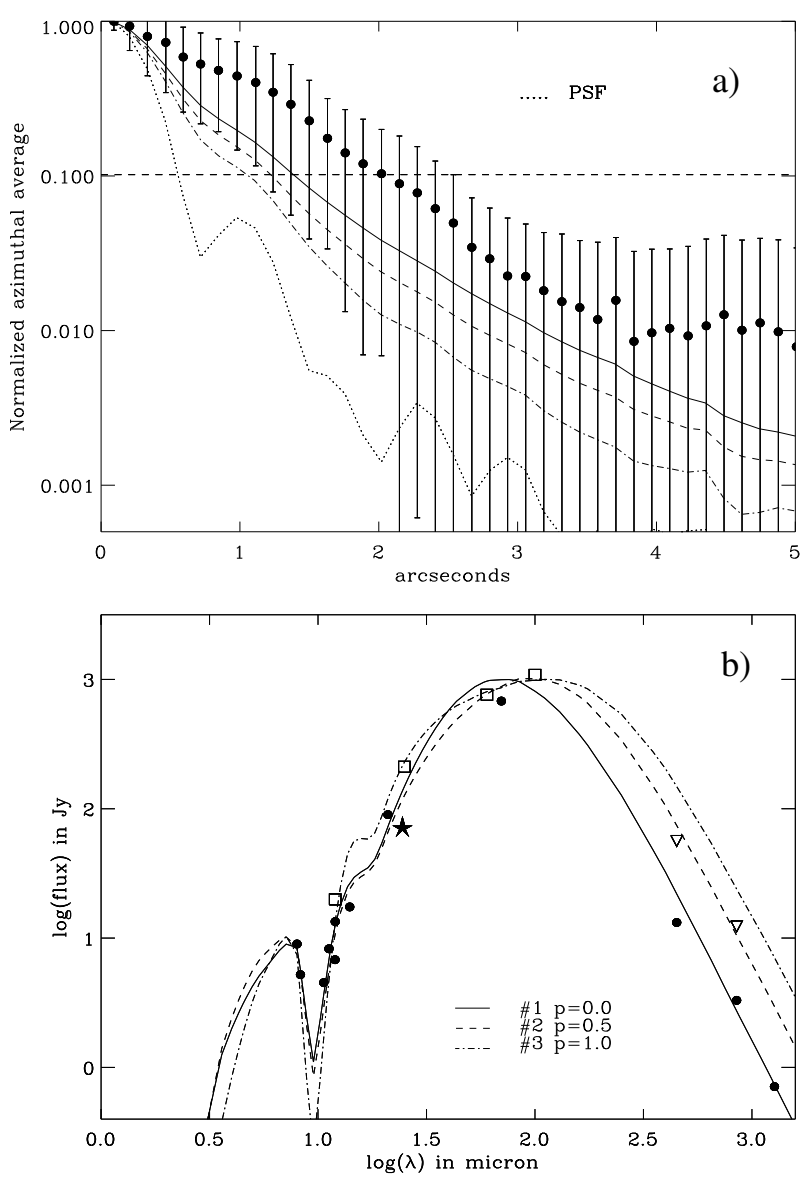

Fig. 24. As Fig. 4, but for AFGL 4029. The SED data is described in Sect. 3.4.1. The best fitting model has a $p=0.0$ radial density profile.

density distribution with a $p=1.0$ power index. Four objects that require steeper $(p>1.0)$ density distributions, viz. AFGL 2591, NGC 2264 IRS1, M8E-IR, S255 IRS3, fail in reproducing either the silicate absorption or the intensity profile. These objects seem to show excess mid-IR flux with respect to spherical models that can only be accounted for if higher bolometric luminosities are adopted, a solution denied by the $24.5 \mu \mathrm{m}$ intensity profile. The MYSO that poses the biggest problem is AFGL 2591. Van der Tak et al. (2000) forwarded the idea that the star's outflow activity is directed nearly along the line-of-sight, and the problems with spherical models encountered here could be a manifestation of this. Schreyer et al. (1997) have argued for NGC 2264 IRS1 that again there is outflow activity along the line-of-sight towards this source. M8E-IR shows evidence of a $\mathrm{CO}$ outflow with the blue and red shifted components projected on top of each other, arguing for a small inclination angle (Simon et al. 1984; see also Linz et al. 2008). If these objects were viewed down the outflow cavities, we would directly view the warm dust at the base of the cavity and perhaps from the accretion disc itself. This extinction free view of the central regions would produce artificially steep laws from spherical models that cannot account for the geometry.

The remaining objects have power indices that are shallower with power indices between $p=0.0$ and $p=0.5$. The best-studied of these objects is IRAS 20126. As discussed in Sect. 3.3.8 this object is a case where there is clear evidence that we are viewing the object close to edge-on. A dark lane appears at wavelengths shorter than $20 \mu \mathrm{m}$. As a result, there will still be significant optical depth at $24.5 \mu \mathrm{m}$ which suppresses the 


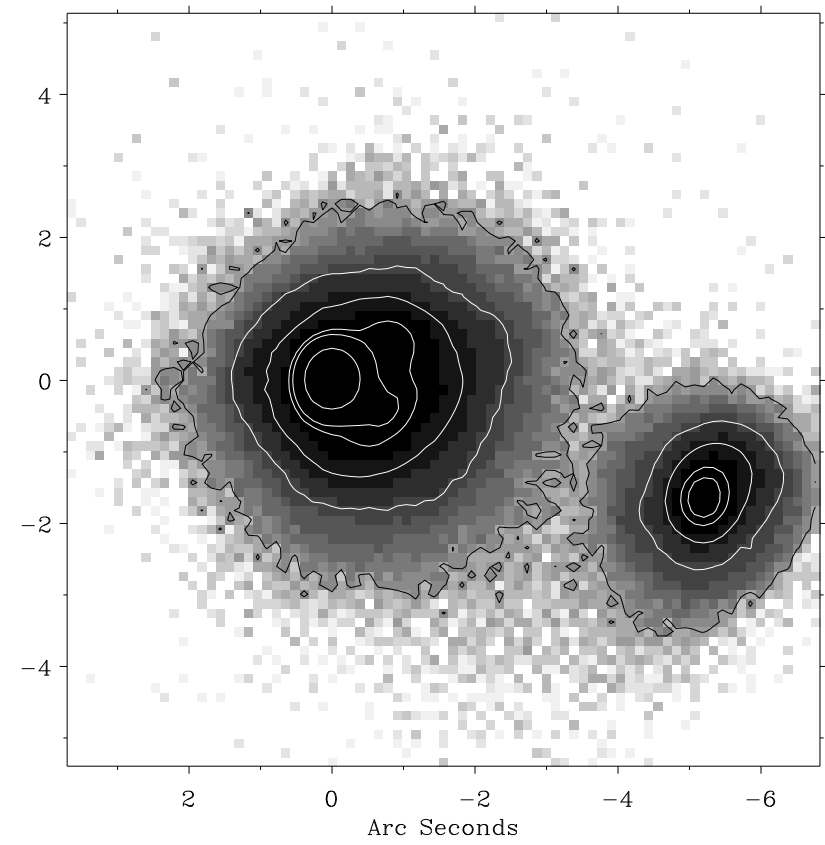

Fig. 25. COMICS $24.5 \mu \mathrm{m}$ image of the AFGL 961 region. Contour levels are at $1 \%, 4 \%, 10 \%, 20 \%, 25 \%$, and $50 \%$ of peak flux density $\left(1.3 \times 10^{2} \mathrm{Jy} \mathrm{arcsec}^{-2}\right)$. North is up, east is to the left.

envelope emission. Instead the weaker, but more extended cavity wall emission becomes significant. This leads to an apparent need for shallower density profiles in our fits.

We therefore conclude that in those MYSO cases where we view the objects at intermediate inclinations, the emission from the envelope dominates. Spherical models are then capable of reproducing simultaneously the $24.5 \mu \mathrm{m}$ intensity profile and the SED. For these cases, radial density profiles with a power index of $p=1.0$ are preferred.

\section{Discussion}

We have presented resolved $24.5 \mu \mathrm{m}$ images of a sample of 14 MYSOs. In most cases, the MYSO is discrete, single (within $<2^{\prime \prime}$ ) and has a circularly symmetric profile on the sky. Simultaneous modelling of the spatial profile and the SED with simple spherical envelope models shows that those objects that can be adequately modelled in this way have radial density powerlaws $n \propto r^{-p}$ with exponent $p=1.0$.

A number of studies have analysed the density structure of MYSO (and UCH II) envelopes by means of 1-D radiative transfer modelling. The approaches consist in reproducing the observed SED and the submm intensity profiles (Mueller et al. 2002; Beuther et al. 2002; Williams et al. 2005) and molecular line emission (van der Tak et al. 2000; Hatchell \& van der Tak 2003). A few important differences exist between these studies and the present one. First, the COMICS observations provide information on scales about ten times smaller, i.e. $1000 \mathrm{AU}$. These scales could correspond to the transition region between the material reservoir (the envelope) and a putative accretion disc. Secondly, the above mentioned studies ease or ignore spectral constraints at wavelengths shorter than $100 \mu \mathrm{m}$. In contrast, our approach is to be more cautious with the IRAS measurements if they cannot be reproduced by any spherical model, but keep the mid-IR and submm measurements. This assumption derives from the likelihood of source confusion and background contamination within the large IRAS beam, and our experience


Fig. 26. As Fig. 4, but for AFGL 961E. The SED data is described in Sect. 3.4.2. The best fitting model has a $p=0.5$ radial density profile.

with Spitzer $70 \mu \mathrm{m}$ fluxes which are often significantly lower than the interpolated IRAS fluxes, even after correction for detector non-linearity effects. In practice, this resulted in somewhat lower bolometric luminosities than usually adopted for our MYSO sample, primarily caused by the luminosity sensitive intensity profile.

As far as the radial density distribution is concerned, we find a preference for $p=1.0$ models, as discussed in Sect. 4. The present analysis shows that radial profiles as steep as $p=2.0$ are incompatible with the observations, and even less steep $p=1.5$ cannot always be justified. Our values are consistent with the ones found by van der Tak et al. (2000) from the submm dust continuum and molecular line emission, albeit on much larger size scales. These authors find a range of 1.0-1.5 for the powerlaw index. Williams et al. (2005) draw a similar conclusion for a large sample of candidate MYSOs. They find an average value of the powerlaw index of $1.3 \pm 0.4$. However, one should note that the models in the latter study require very high optical depths (generally optically thick at $100 \mu \mathrm{m}$ ), which is a factor of a few to an order of magnitude larger than derived by us. It goes to show that the DUSTY models presented by Williams et al. would probably never fit the silicate feature nor the mid-IR part of the SED, and indeed they do not attempt to fit the mid-IR nor the far-IR data points. Mueller et al. (2002) claim a value for the power index "p" which is consistent with the previous two but slightly higher: $1.8 \pm 0.4$. Importantly, they argue that the van der Tak (2000) results would favour higher values for the power index if they had convolved their models with the actual 


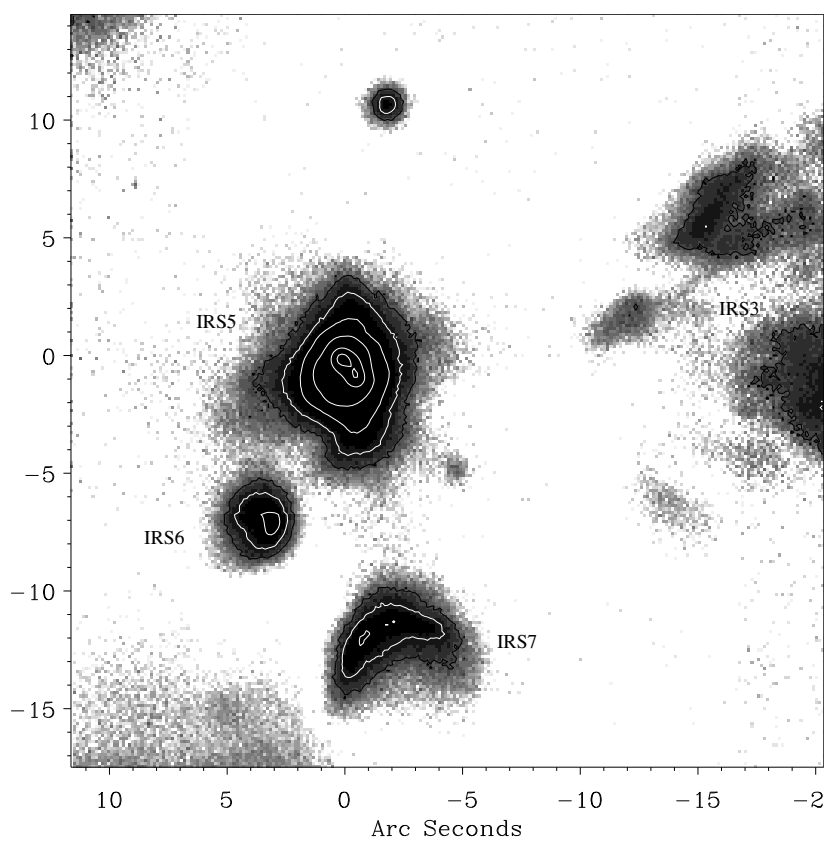

Fig. 27. COMICS $24.5 \mu \mathrm{m}$ mosaic of the W3 region. Contour levels are at $1 \%, 2 \%, 5 \%, 15 \%, 50 \%$, and $85 \%$ of peak flux density $\left(3.8 \times 10^{2} \mathrm{Jy} \mathrm{arcsec}^{-2}\right)$. North is up, east is to the left.

telescope beam instead of a Gaussian profile. Finally, Beuther et al. (2002) claim $1.6 \pm 0.5$ from powerlaw fits to the inner $32^{\prime \prime}$ of their resolved $1.2 \mathrm{~mm}$ images. In summary, the far-IR and submm studies generally prefer somewhat steeper density profiles than the ones derived from $24.5 \mu \mathrm{m}$ images, recalling the caveats regarding the van der Tak et al. (2000) and Williams et al. (2005) results. This apparent inconsistency can however be brought into agreement when allowing for the different spatial scales probed.

On small angular scales, various studies in the (sub)mm find evidence for shallower density distributions or flat intensity distributions, which are suggested to be due to an unresolved core or possibly a collection of cores. For example, Hatchell et al. (2000) find better model fits to their submm intensity profiles of a number of UCH II regions, when they include an unresolved, high optical depth, central core. Beuther et al. (2002) describe the $1.2 \mathrm{~mm}$ intensity profiles of a large sample of massive cores with models that require an unresolved, inner, constant intensity distribution with radii between 2000-60000 AU. Van der Tak et al. $(1999,2000)$ conclude from their interferometric mm data (probing $\sim 1000 \mathrm{AU}$ scales) that the detected emission is caused by a compact structure with an estimated angular size of $0.3^{\prime \prime}$, and which is different from the larger scale spherical envelope structure.

Compact substructures thus seem to be required in order to explain the (sub)mm observations. Flat intensity distributions are suggestive of an optically thick dust component, such as a dense shell or a disc (van der Tak et al. 2000), or a collection of subcores created by the ongoing fragmentation of the large-scale molecular core (Beuther et al. 2002). In the latter case, the low angular resolution single dish submm observations would find the integrated emission of the various subcores to be equivalent to a shallow or a flat density distribution. It can therefore be argued that the flattening of the radial density distribution seen in the submm close to the unresolved central source is consistent with the relatively shallow density distribution as traced by our high-resolution $24.5 \mu \mathrm{m}$ images.


Fig. 28. As Fig. 4, but for W3 IRS 5. The SED data is described in Sect. 3.4.3. The best fitting model has a $p=1.0$ radial density profile.

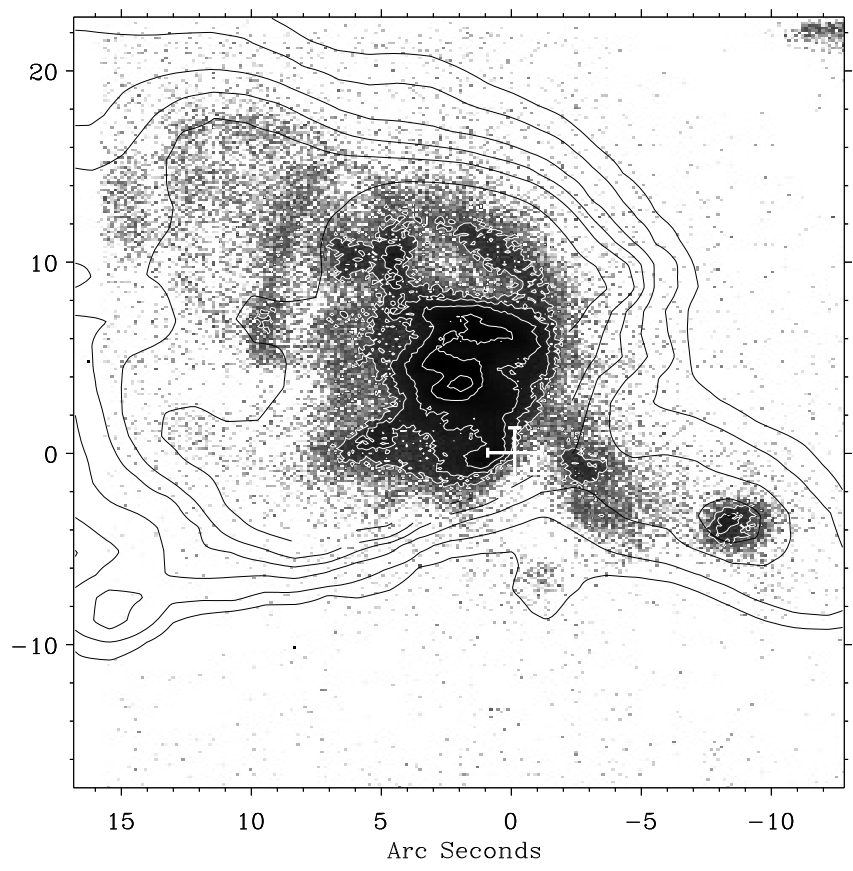

Fig. 29. COMICS $24.5 \mu \mathrm{m}$ mosaic of the Cep A region. Dark contours represent Spitzer $8 \mu \mathrm{m}$ emission. Centre of the coordinate system corresponds to the phase centre of the VLA $7 \mathrm{~mm}$ continuum map in Jiménez-Serra et al. (2007): $\alpha=22^{\mathrm{h}} 56^{\mathrm{m}} 18.0^{\mathrm{s}}, \delta=62^{\circ} 01^{\prime} 49.5^{\prime \prime}$ (indicated by a white cross). Contour levels of the $24.5 \mu \mathrm{m}$ emission are at $10 \%, 15 \%, 25 \%, 50 \%$, and $80 \%$ of peak flux density $\left(2.7 \times 10^{1} \mathrm{Jy} \operatorname{arcsec}^{-2}\right)$. North is up, east is to the left. 
Table 5. Overview of the quality of the model fits. The second column gives the power index of the best-fitting radial density distribution. A colon is added to uncertain values. A tickmark indicates a reasonable correspondence between model and observations; an " $x$ " means no correspondence, and ".." indicates that not enough data or only data of insufficient quality is available.

\begin{tabular}{|c|c|c|c|c|c|c|c|}
\hline MYSO & $p$ & $\begin{array}{l}4-8 \\
\mu \mathrm{m}\end{array}$ & $\begin{array}{l}9.7 \\
\mu \mathrm{m}\end{array}$ & $\begin{array}{l}24.5 \\
\mu \mathrm{m}\end{array}$ & $\begin{array}{l}100 \\
\mu \mathrm{m}\end{array}$ & $\overline{\mathrm{mm}}$ & $\begin{array}{c}\text { Intensity } \\
\text { profile }\end{array}$ \\
\hline S140 IRS1 & 1.0 & $\mathrm{x}$ & $\sqrt{ }$ & $\sqrt{ }$ & $\sqrt{ }$ & $\sqrt{ }$ & $\sqrt{ }$ \\
\hline AFGL2136 & 1.0 & $\mathrm{x}$ & $\sqrt{ }$ & $\sqrt{ }$ & .. & $\sqrt{ }$ & $\sqrt{ }$ \\
\hline AFGL 5180 & 1.0 & .. & 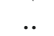 & $\sqrt{ }$ & $\sqrt{ }$ & $\sqrt{ }$ & $\sqrt{ }$ \\
\hline Mon R2 IRS3 & 1.0 & $\mathrm{x}$ & $\sqrt{ }$ & $\sqrt{ }$ & .. & $\sqrt{ }$ & $\sqrt{ }$ \\
\hline W3 IRS5 & 1.0: & $\mathrm{x}$ & $\sqrt{ }$ & $\sqrt{ }$ & $\mathrm{x}$ & $\sqrt{ }$ & $\sqrt{ }$ \\
\hline M8E-IR & 1.25 & $\mathrm{x}$ & $\mathrm{x}$ & $\sqrt{ }$ & .. & $\sqrt{ }$ & $\sqrt{ }$ \\
\hline S255 IRS3 & 1.25 & $\mathrm{x}$ & $\sqrt{ }$ & $\sqrt{ }$ & .. & $\sqrt{ }$ & $\mathrm{x}$ \\
\hline AFGL 2591 & 1.5 & $\mathrm{x}$ & $\mathrm{x}$ & $\sqrt{ }$ & .. & $\sqrt{ }$ & $\mathrm{x}$ \\
\hline NGC 2264 IRS1 & 1.5 & $\mathrm{x}$ & $\mathrm{x}$ & $\sqrt{ }$ & 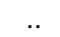 & $\sqrt{ }$ & $\sqrt{ }$ \\
\hline IRAS 20126 & 0.0 & $\mathrm{x}$ & 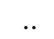 & $\sqrt{ }$ & $\sqrt{ }$ & $\sqrt{ }$ & $\sqrt{ }$ \\
\hline AFGL 437S & 0.0 & .. & $\sqrt{ }$ & $\sqrt{ }$ & . & $\sqrt{ }$ & $\sqrt{ }$ \\
\hline AFGL 4029 & 0.0 : & .. & .. & $\sqrt{ }$ & $\sqrt{ }$ & $\sqrt{ }$ & $\sqrt{ }$ \\
\hline AFGL 961E & 0.5 : & $\sqrt{ }$ & $\sqrt{ }$ & $\sqrt{ }$ & $\sqrt{ }$ & $\sqrt{ }$ & $\sqrt{ }$ \\
\hline
\end{tabular}

The images are generally dominated by one single, resolved principal source, identified with the MYSO, which has a circular appearance on the sky. Only in a small minority of cases do we find more than one condensation on scales $\lesssim 2$ ". This observation goes against the idea of fragmentation as the main cause of the flattening of the inner density profile. Rodón et al. (2008) present $0.35^{\prime \prime}$ resolution $\mathrm{mm}$ images of the near-IR double source W3 IRS5, in which the dominant component does not resolve into multiple $\mathrm{mm}$ sources. Given that this source is the most luminous source in our sample, any fragmentation into a cluster of protostars would be expected in particular in this source, but this is not observed. Instead, it seems that the $24.5 \mu \mathrm{m}$ could indicate that it is the density distribution of the ambient core material itself that actually flattens out at scales of an arcsecond. An alternative explanation why this could be so is rotation. The density structure for a rotating and infalling envelope (TSC envelopes, Terebey et al. 1984) becomes on average significantly shallower within the so-called centrifugal radius, i.e. where rotational motion dominates over infall. The evolution of the density distribution from relatively steep on large scales as seen by single-dish the (sub)mm observations to shallow on smaller scales at shorter wavelengths could be a manifestation of this effect.

The inability of models with spherical and smooth density distributions to reproduce the near-IR and mid-IR part of the SED was demonstrated again in the analysis presented in this paper. The development of more sophisticated models (e.g. Yorke \& Sonnhalter 2002; Whitney et al. 2003; Indebetouw et al. 2006) is driven in part to reproduce this emission. Radiative transfer models that incorporate the mentioned rotating TSC envelopes and implement a flared equatorial accretion disc and a bipolar outflow cavity are presented in Whitney et al. (2003). The geometrical features are inspired mainly by detailed observations of low-mass class I YSOs (Whitney et al. 2003; see also Tobin et al. 2008) and may therefore be considered more realistic than the simple spherical models we have adopted here.

Recent work has applied the more sophisticated envelope models to MYSO SEDs and concluded that high-mass star formation proceeds similar to low-mass star formation (e.g. De Buizer et al. 2008; Fazal et al. 2007). Such work is becoming popular especially after the publication of an extensive grid of SEDs (Robitaille et al. 2006) that are based on the Whitney et al. models. The rationale behind the Robitaille et al. SED grid is that high-mass stars may form similarly to low-mass stars, or at least that the SEDs of MYSOs are determined by similar geometrical structures as found in low-mass objects. Inferring 2D (or 3D) information from SED fits only is prone to be subjective and misleading. The quoted conclusion therefore that massive SF is similar to low-SF based on SED fits to such models is a circular argument. What the Whitney et al. models in particular show is that one should be careful interpreting SEDs, without any independent additional data. Large scale spatial information of the dust emission in MYSOs can be retrieved from the singledish (sub)mm studies previously mentioned, intermediate scales can be probed in the mid-IR as our study presented here, and with (sub)mm interferometry. Finally, scales down to $100 \mathrm{AU}$ in the mid-IR can be reached with mid-IR interferometry using e.g. the VLTI (de Wit et al. 2007; Linz et al. 2008). Eventually the question of how a massive star forms can only be settled with such spatially resolved information.

\section{Conclusions}

We have presented a study of resolved $24.5 \mu \mathrm{m}$ images of 14 massive star forming regions. The images probe linear size scales of $1000 \mathrm{AU}$. Emission at $24.5 \mu \mathrm{m}$ is dominated by the MYSOs in these regions. They are discrete sources and most have a circular profile to first order. In three cases we find multiple condensations embedded in a larger mid-IR envelope. Various regions display extended, diffuse emission. This emission is associated with UCH II regions (e.g. Mon R2, AFGL 437, AFGL 2591) in which case the dust is heated by Ly $\alpha$ within the ionized zone. Shock excited material (e.g. S 140) also seems to produce diffuse emission at $24.5 \mu \mathrm{m}$.

Simple 1-D spherical model fits to the MYSO $24.5 \mu \mathrm{m}$ spatial profile and SED show that radial density powerlaws of the form $n=n_{0}\left(r / r_{\text {subl }}\right)^{-p}$ with a power $p=1.0$ are preferred. When there is evidence that we are viewing the MYSO either face-on down the outflow cavity or edge-on through a torus we find steeper or shallower density laws respectively. These density laws are more likely to be due to the inadequacies of the spherical models in these cases than a true representation of a different density law, and we expect that $p=1.0$ also applies there to.

We find that the spatial profile of the dust emission on scales of $1000 \mathrm{AU}$ is shallower than that from larger $10000 \mathrm{AU}$ scales probed by (sub)mm dust emission. Inner flattenings seen in the submm are consistent with our results here. This flattening is not likely to be due to fragmentation of the core, but due to the actual distribution of the emitting material. This is supported by the relatively small multiplicity of condensations seen on sub-arcsecond scales. The continuous flattening from large to small scales could be the manifestation of rotation, but this requires further study. The application of more sophisticated, multi-dimensional models in relation to the data presented here and at even higher resolution using mid-IR interferometric observations will be the subject of a future paper.

Acknowledgements. R.D.O. is grateful for the support from the Leverhulme Trust for awarding a Research Fellowship. The authors would like to thank I. Jiménez-Serra and E. R. Parkin for fruitful discussions, and an anonymous referee for valuable comments. The version of the ISO data presented in this paper correspond to the Highly Processed Data Product (HPDP) by W. F. Frieswijk et al., available for public use in the ISO Data Archive. This research has made use of the SIMBAD database and the VizieR catalogue access tool, operated at CDS, Strasbourg, France. This research has made use of NASA's Astrophysics 
Data System. We acknowledge the use of the RMS database, that can be found at URL www . ast. leeds. ac. uk/RMS/.

\section{References}

Alvarez, C., \& Hoare, M. G. 2005, A\&A, 440, 569

Alvarez, C., Hoare, M., Glindemann, A., \& Richichi, A. 2004, A\&A, 427, 505 Aspin, C. 1998, A\&A, 335, 1040

Becker, W., \& Fenkart, R. 1971, A\&AS, 4, 241

Beckwith, S., Evans, II, N. J., Becklin, E. E., \& Neugebauer, G. 1976, ApJ, 208, 390

Beichman, C. A., Becklin, E. E., \& Wynn-Williams, C. G. 1979, ApJ, 232, L47

Beltrán, M. T., Brand, J., Cesaroni, R., et al. 2006, A\&A, 447, 221

Beuther, H., Schilke, P., Menten, K. M., et al. 2002, ApJ, 566, 945

Campbell, M. F., Butner, H. M., Harvey, P. M., et al. 1995, ApJ, 454, 831

Castelaz, M. W., Grasdalen, G. L., Hackwell, J. A., Capps, R. W., \& Thompson, D. 1985, AJ, 90, 1113

Cesaroni, R., Felli, M., Testi, L., Walmsley, C. M., \& Olmi, L. 1997, A\&A, 325, 725

Cesaroni, R., Felli, M., Jenness, T., et al. 1999, A\&A, 345, 949

Cesaroni, R., Neri, R., Olmi, L., et al. 2005, A\&A, 434, 1039

Chini, R., Kreysa, E., Mezger, P. G., \& Gemuend, H.-P. 1986a, A\&A, 154, L8

Chini, R., Kruegel, E., \& Kreysa, E. 1986b, A\&A, 167, 315

Churchwell, E., Wolfire, M. G., \& Wood, D. O. S. 1990, ApJ, 354, 247

Claussen, M. J., Gaume, R. A., Johnston, K. J., \& Wilson, T. L. 1994, ApJ, 424, L41

Cohen, M. 1973, ApJ, 185, L75+

Cohen, M., Witteborn, F. C., Walker, R. G., Bregman, J. D., \& Wooden, D. H. 1995, AJ, 110, 275

Cohen, M., Walker, R. G., Carter, B., et al. 1999, AJ, 117, 1864

Crampton, D., \& Fisher, W. A. 1974, Spectroscopic observations of stars in HII regions Crampton and W. A. Fisher (Victoria: National Research Council of Canada, Radio and Electrical Engineering Division, Astrophysics Branch), 12

Curiel, S., Ho, P. T. P., Patel, N. A., et al. 2006, ApJ, 638, 878

Dale, D. A., Gil de Paz, A., Gordon, K. D., et al. 2007, ApJ, 655, 863

De Buizer, J. M. 2007, ApJ, 654, L147

De Buizer, J. M., Piña, R. K., \& Telesco, C. M. 2000, ApJS, 130, 437

De Buizer, J. M., Osorio, M., \& Calvet, N. 2005a, ApJ, 635, 452

De Buizer, J. M., Radomski, J. T., Telesco, C. M., \& Piña, R. K. 2005b, ApJS, 156,179

de Graauw, T., Haser, L. N., Beintema, D. A., et al. 1996, A\&A, 315, L49

de Wit, W. J., Hoare, M. G., Oudmaijer, R. D., \& Mottram, J. C. 2007, ApJ, 671, L169

de Wit, W. J., Oudmaijer, R. D., Fujiyoshi, T., et al. 2008, ApJ, 685, L75

Deharveng, L., Zavagno, A., Cruz-Gonzalez, I., et al. 1997, A\&A, 317, 459

Dent, W. R. F., Matthews, H. E., \& Ward-Thompson, D. 1998, MNRAS, 301, 1049

Di Francesco, J., Johnstone, D., Kirk, H., MacKenzie, T., \& Ledwosinska, E. 2008, ApJS, 175, 277

Draine, B. T., \& Lee, H. M. 1984, ApJ, 285, 89

Evans, II, N. J., Beckwith, S., \& Blair, G. N. 1977, ApJ, 217, 448

Evans, II, N. J., Slovak, M. H., Becklin, E. E., et al. 1981, ApJ, 244, 115

Evans, II, N. J., Mundy, L. G., Kutner, M. L., \& Depoy, D. L. 1989, ApJ, 346, 212

Faison, M., Churchwell, E., Hofner, P., et al. 1998, ApJ, 500, 280

Fazal, F. M., Sridharan, T. K., Qiu, K., et al. 2007, ArXiv e-prints

Forrest, W. J., \& Shure, M. A. 1986, ApJ, 311, L81

Gear, W. K., Robson, E. I., \& Griffin, M. J. 1988, MNRAS, 231, 55P

Gezari, D. Y., Pitts, P. S., \& Schmitz, M. 1999, VizieR Online Data Catalog, 2225,0

Ghosh, S. K., Iyengar, K. V. K., Karnik, A. D., et al. 2000, BASI, 28, 515

Giannakopoulou, J., Mitchell, G. F., Hasegawa, T. I., Matthews, H. E., \& Maillard, J.-P. 1997, ApJ, 487, 346

Gibb, A. G., \& Hoare, M. G. 2007, MNRAS, 380, 246

Gómez, J. F., Sargent, A. I., Torrelles, J. M., et al. 1999, ApJ, 514, 287

Greenhill, L. J., Gezari, D. Y., Danchi, W. C., et al. 2004, ApJ, 605, L57

Guertler, J., Henning, T., Kruegel, E., \& Chini, R. 1991, A\&A, 252, 801

Hackwell, J. A., Grasdalen, G. L., \& Gehrz, R. D. 1982, ApJ, 252, 250

Harvey, P. M., Campbell, M. F., \& Hoffmann, W. F. 1977, ApJ, 215, 151

Harvey, P. M., Butner, H. M., Colomé, C., Di Francesco, J., \& Smith, B. J. 2000, ApJ, 534, 846

Hatchell, J., \& van der Tak, F. F. S. 2003, A\&A, 409, 589

Hatchell, J., Fuller, G. A., Millar, T. J., Thompson, M. A., \& Macdonald, G. H. 2000, A\&A, 357, 637

Hayashi, M., Hasegawa, T., Omodaka, T., Hayashi, S. S., \& Miyawaki, R. 1987, ApJ, 312, 327

Henning, T., Pfau, W., \& Altenhoff, W. J. 1990, A\&A, 227, 542
Henning, T., Chini, R., \& Pfau, W. 1992, A\&A, 263, 285

Henning, T., Schreyer, K., Launhardt, R., \& Burkert, A. 2000, A\&A, 353, 211

Herbst, W., \& Racine, R. 1976, AJ, 81, 840

Hoare, M. G. 2006, ApJ, 649, 856

Hoare, M. G., \& Franco, J. 2007, Massive Star Formation (Diffuse Matter from Star Forming Regions to Active Galaxies), 61

Hoare, M. G., Roche, P. F., \& Glencross, W. M. 1991, MNRAS, 251, 584

Hoare, M. G., Kurtz, S. E., Lizano, S., Keto, E., \& Hofner, P. 2007, in Protostars and Planets V, ed. B. Reipurth, D. Jewitt, \& K. Keil, 181

Hofner, P., Cesaroni, R., Olmi, L., et al. 2007, A\&A, 465, 197

Howard, E. M., Pipher, J. L., \& Forrest, W. J. 1994, ApJ, 425, 707

Howell, R. R., McCarthy, D. W., \& Low, F. J. 1981, ApJ, 251, L21

Hughes, V. A., \& Wouterloot, J. G. A. 1984, ApJ, 276, 204

Imai, H., Kameya, O., Sasao, T., et al. 2000, ApJ, 538, 751

Indebetouw, R., Whitney, B. A., Johnson, K. E., \& Wood, K. 2006, ApJ, 636, 362

Itoh, Y., Tamura, M., Suto, H., et al. 2001, PASJ, 53, 495

Ivezic, Z., \& Elitzur, M. 1997, MNRAS, 287, 799

Jaffe, D. T., Davidson, J. A., Dragovan, M., \& Hildebrand, R. H. 1984, ApJ, 284, 637

Jenness, T., Scott, P. F., \& Padman, R. 1995, MNRAS, 276, 1024

Jiang, Z., Tamura, M., Hoare, M. G., et al. 2008, ApJ, 673, L175

Jiménez-Serra, I., Martín-Pintado, J., Rodríguez-Franco, A., et al. 2007, ApJ, 661, L187

Kastner, J. H., Weintraub, D. A., \& Aspin, C. 1992, ApJ, 389, 357

Kastner, J. H., Weintraub, D. A., Snell, R. L., et al. 1994, ApJ, 425, 695

Kataza, H., Okamoto, Y., Takubo, S., et al. 2000, in SPIE Conf. Ser. 4008, ed. M. Iye \& A. F. Moorwood, 1144

Kessler, M. F., Steinz, J. A., Anderegg, M. E., et al. 1996, A\&A, 315, L27

Klein, R., Posselt, B., Schreyer, K., Forbrich, J., \& Henning, T. 2005, ApJS, 161, 361

Kurtz, S., Churchwell, E., \& Wood, D. O. S. 1994, ApJS, 91, 659

Lada, C. J., \& Gautier, III, T. N. 1982, ApJ, 261, 161

Lada, C. J., Thronson, Jr., H. A., Smith, H. A., Schwartz, P. R., \& Glaccum, W. 1984, ApJ, 286, 302

Ladd, E. F., Deane, J. R., Sanders, D. B., \& Wynn-Williams, C. G. 1993, ApJ, 419,186

Lenzen, R., Hodapp, K.-W., \& Reddmann, T. 1984a, A\&A, 137, 365

Lenzen, R., Hodapp, K.-W., \& Solf, J. 1984b, A\&A, 137, 202

Lester, D. F., Harvey, P. M., Joy, M., \& Ellis, Jr., H. B. 1986, ApJ, 309, 80

Linz, H., Henning, T., Stecklum, B., et al. 2008, in Massive Star Formation: Observations Confront Theory, ed. H. Beuther, H. Linz, \& T. Henning, ASP Conf. Ser., 387, 132

Longmore, S. N., Burton, M. G., Minier, V., \& Walsh, A. J. 2006, MNRAS, 369 , 1196

Marsh, K. A., Bloemhof, E. E., Koerner, D. W., \& Ressler, M. E. 2001, ApJ, 548,861

Martín-Pintado, J., Jiménez-Serra, I., Rodríguez-Franco, A., Martín, S., \& Thum, C. 2005, ApJ, 628, L61

Massi, M., Felli, M., \& Simon, M. 1985, A\&A, 152, 387

Mathis, J. S., Rumpl, W., \& Nordsieck, K. H. 1977, ApJ, 217, 425

Meakin, C. A., Hines, D. C., \& Thompson, R. I. 2005, ApJ, 634, 1146

Menten, K. M., \& van der Tak, F. F. S. 2004, A\&A, 414, 289

Mezger, P. G., Chini, R., Kreysa, E., Wink, J. E., \& Salter, C. J. 1988, A\&A, 191,44

Minchin, N. R., Ward-Thompson, D., \& White, G. J. 1995, A\&A, 298, 894

Minier, V., Burton, M. G., Hill, T., et al. 2005, A\&A, 429, 945

Moffat, A. F. J., Jackson, P. D., \& Fitzgerald, M. P. 1979, A\&AS, 38, 197

Mottram, J. C., Hoare, M. G., Lumsden, S. L., et al. 2007, A\&A, 476, 1019

Mueller, K. E., Shirley, Y. L., Evans, II, N. J., \& Jacobson, H. R. 2002, ApJS, 143,469

Nakano, M., Sugitani, K., \& Morita, K. 2003, PASJ, 55, 1

Ossenkopf, V., \& Henning, T. 1994, A\&A, 291, 943

Patel, N. A., Curiel, S., Sridharan, T. K., et al. 2005, Nature, 437, 109

Peretto, N., André, P., \& Belloche, A. 2006, A\&A, 445, 979

Preibisch, T., Balega, Y. Y., Schertl, D., Smith, M. D., \& Weigelt, G. 2001, A\&A, 378,539

Preibisch, T., Balega, Y. Y., Schertl, D., \& Weigelt, G. 2002, A\&A, 392, 945

Preibisch, T., Balega, Y. Y., Schertl, D., \& Weigelt, G. 2003, A\&A, 412, 735

Rayner, J., \& McLean, I. 1987, in Infrared astronomy with arrays, ed. C. G. Wynn-Williams, \& E. E. Becklin, 272

Richardson, K. J., White, G. J., Gee, G., et al. 1985, MNRAS, 216, 713

Robitaille, T. P., Whitney, B. A., Indebetouw, R., Wood, K., \& Denzmore, P. 2006, ApJS, 167, 256

Rodón, J. A., Beuther, H., Megeath, S. T., \& van der Tak, F. F. S. 2008, A\&A, 490,213

Saito, H., Saito, M., Moriguchi, Y., \& Fukui, Y. 2006, PASJ, 58, 343

Saito, H., Saito, M., Sunada, K., \& Yonekura, Y. 2007, ApJ, 659, 459 
Scarrott, S. M. \& Warren-Smith, R. F. 1989, MNRAS, 237, 995

Schreyer, K., Helmich, F. P., van Dishoeck, E. F., \& Henning, T. 1997, A\&A, 326,347

Schreyer, K., Stecklum, B., Linz, H., \& Henning, T. 2003, ApJ, 599, 335

Shepherd, D. S., Yu, K. C., Bally, J., \& Testi, L. 2000, ApJ, 535, 833

Shuping, R. Y., Morris, M., \& Bally, J. 2004, AJ, 128, 363

Simon, M., Cassar, L., Felli, M., et al. 1984, ApJ, 278, 170

Simon, M., Peterson, D. M., Longmore, A. J., Storey, J. W. V., \& Tokunaga,

A. T. 1985, ApJ, 298, 328

Simon, T., \& Dyck, H. M. 1977, AJ, 82, 725

Sloan, G. C., Kraemer, K. E., Price, S. D., \& Shipman, R. F. 2003, ApJS, 147, 379

Snell, R. L., \& Bally, J. 1986, ApJ, 303, 683

Snell, R. L., Huang, Y.-L., Dickman, R. L., \& Claussen, M. J. 1988, ApJ, 325, 853

Sridharan, T. K., Williams, S. J., \& Fuller, G. A. 2005, ApJ, 631, L73

Su, Y.-N., Liu, S.-Y., Chen, H.-R., Zhang, Q., \& Cesaroni, R. 2007, ApJ, 671, 571

Tamura, M., Gatley, I., Joyce, R. R., et al. 1991, ApJ, 378, 611

Tatebe, K., Hale, D. D. S., Wishnow, E. H., \& Townes, C. H. 2007, ApJ, 658, L103

Terebey, S., Shu, F. H., \& Cassen, P. 1984, ApJ, 286, 529

Thompson, M. A., Hatchell, J., Walsh, A. J., MacDonald, G. H., \& Millar, T. J. 2006, A\&A, 453, 1003

Thompson, R. I., Corbin, M. R., Young, E., \& Schneider, G. 1998, ApJ, 492, L177

Thronson, Jr., H. A., Gatley, I., Harvey, P. M., Sellgren, K., \& Werner, M. W. 1980, ApJ, 237, 66

Thronson, Jr., H. A., Lada, C. J., Smith, H. A., et al. 1983, ApJ, 271, 625

Tobin, J. J., Hartmann, L., Calvet, N., \& D’Alessio, P. 2008, ApJ, 679, 1364

Tofani, G., Felli, M., Taylor, G. B., \& Hunter, T. R. 1995, A\&AS, 112, 299
Torrelles, J. M., Gomez, J. F., Anglada, G, et al. 1992, ApJ, 392, 616

van der Tak, F. F. S., van Dishoeck, E. F., Evans, II, N. J., Bakker, E. J., \& Blake, G. A. 1999, ApJ, 522, 991

van der Tak, F. F. S., van Dishoeck, E. F., Evans, II, N. J., \& Blake, G. A. 2000, ApJ, 537, 283

van der Tak, F. F. S., Tuthill, P. G., \& Danchi, W. C. 2005, A\&A, 431, 993

Trinidad, M. A., Curiel, S., Cantó, J., et al. 2003, ApJ, 589, 386

Walker, C. K., Adams, F. C., \& Lada, C. J. 1990, ApJ, 349, 515

Walker, M. F. 1956, ApJS, 2, 365

Ward-Thompson, D., Zylka, R., Mezger, P. G., \& Sievers, A. W. 2000, A\&A, 355,1122

Weigelt, G., Balega, Y. Y., Preibisch, T., Schertl, D., \& Smith, M. D. 2002, A\&A, 381,905

Weintraub, D. A., \& Kastner, J. H. 1996, ApJ, 458, 670

Whitney, B. A., Wood, K., Bjorkman, J. E., \& Wolff, M. J. 2003, ApJ, 591, 1049

Williams, S. J., Fuller, G. A., \& Sridharan, T. K. 2005, A\&A, 434, 257

Willner, S. P., Gillett, F. C., Herter, T. L., et al. 1982, ApJ, 253, 174

Wilson, T. L., Boboltz, D. A., Gaume, R. A., \& Megeath, S. T. 2003, ApJ, 597, 434

Wolfire, M. G., \& Churchwell, E. 1994, ApJ, 427, 889

Wynn-Williams, C. G. 1982, ARA\&A, 20, 587

Wynn-Williams, C. G., Becklin, E. E., \& Neugebauer, G. 1972, MNRAS, 160, 1

Wynn-Williams, C. G., Forster, J. R., Welch, W. J., et al. 1977, ApJ, 211, L89

Wynn-Williams, C. G., Becklin, E. E., Beichman, C. A., Capps, R., \& Shakeshaft, J. R. 1981, ApJ, 246, 801

Yorke, H. W., \& Sonnhalter, C. 2002, ApJ, 569, 846

Zapata, L. A., Rodríguez, L. F., \& Kurtz, S. E. 2001, Rev. Mex. Astron. Astrofis., 37,83

Zavagno, A., Lagage, P. O., \& Cabrit, S. 1999, A\&A, 344, 499

Zubko, V. G., Mennella, V., Colangeli, L., \& Bussoletti, E. 1996, MNRAS, 282, 1321 